\title{
Vps34 regulates myofibril proteostasis to prevent hypertrophic cardiomyopathy
}

\author{
Hirotaka Kimura, ${ }^{1,2}$ Satoshi Eguchi, ${ }^{2}$ Junko Sasaki, ${ }^{2}$ Keiji Kuba, ${ }^{3}$ Hiroki Nakanishi, \\ Shunsuke Takasuga, ${ }^{2}$ Masakazu Yamazaki, ${ }^{4}$ Akiteru Goto, ${ }^{5}$ Hiroyuki Watanabe, ${ }^{6}$ Hiroshi Itoh, ${ }^{6}$ \\ Yumiko Imai, ${ }^{3}$ Akira Suzuki, ${ }^{7}$ Noboru Mizushima, ${ }^{8}$ and Takehiko Sasaki ${ }^{1,2}$ \\ ${ }^{1}$ Research Center for Biosignaling, Department of ${ }^{2}$ Medical Biology, ${ }^{3}$ Biological Informatics and Experimental Therapeutics \\ Pathology, ${ }^{4}$ Cell Biology and Morphology, ${ }^{5}$ Cellular and Organ Pathology, and ${ }^{6}$ Cardiovascular and Respiratory Medicine, \\ Akita University Graduate School of Medicine, Akita, Japan. D. Department of Molecular Genetics, Division of Cancer \\ Genetics, Kobe University Graduate School of Medicine, Kobe, Japan. ${ }^{8}$ Department of Biochemistry and Molecular Biology, \\ Graduate School and Faculty of Medicine, University of Tokyo, Tokyo, Japan.
}

Hypertrophic cardiomyopathy (HCM) is a common heart disease with a prevalence of 1 in 500 in the general population. Several mutations in genes encoding cardiac proteins have been found in HCM patients, but these changes do not predict occurrence or prognosis and the molecular mechanisms underlying HCM remain largely elusive. Here we show that cardiac expression of vacuolar protein sorting 34 (Vps34) is reduced in a subset of HCM patients. In a mouse model, muscle-specific loss of Vps34 led to HCM-like manifestations and sudden death. Vps34-deficient hearts exhibited abnormal histopathologies, including myofibrillar disarray and aggregates containing $\alpha \mathrm{B}$-crystallin (CryAB). These features result from a block in the ESCRT-mediated proteolysis that normally degrades K63-polyubiquitinated CryAB. CryAB deposition was also found in myocardial specimens from a subset of HCM patients whose hearts showed decreased Vps34. Our results identify disruption of the previously unknown Vps34-CryAB axis as a potentially novel etiology of HCM.

Conflict of interest: The authors have declared that no conflict of interest exists.

Submitted: July 12, 2016 Accepted: November 22, 2016 Published: January 12, 2017

Reference information: JCI Insight. 2017;2(1):e89462. doi:10.1172/jci.insight.89462.

\section{Introduction}

Global statistics indicate that approximately 17.5 million people die of heart diseases every year, representing $30 \%$ of the total death rate worldwide (1). Two major classes of heart diseases are ischemic cardiomyopathy (ICM) and nonischemic cardiomyopathy (NICM), with hypertrophic cardiomyopathy (HCM) being a key member of the latter class. HCM, which is defined by the presence of ventricular hypertrophy in the European Society of Cardiology (ESC) guidelines on diagnosis (2), is the most frequent cause of sudden death in young people and can lead to functional disability from heart failure (3). Mutations in genes encoding proteins of the cardiac sarcomere, such as $\beta$-myosin heavy chain (MYH7) and myosin-binding protein C (MYBPC3), have been identified in about 50\% of HCM cases (4). However, there is much phenotypic heterogeneity in HCM (i.e., differences in symptom onset, disease progression and severity) even within families in which affected individuals all carry the same mutation (3-5). It is also known that some cases with HCM progress to dilated cardiomyopathy, another type of NICM characterized by ventricular dilation and impaired systolic function (6). Hence, it is widely argued that knowing the causative mutation alone in an HCM case is of limited utility in determining patient prognosis. Further stratification of $\mathrm{HCM}$ patients based on their underlying biological (in addition to genetic) alterations may provide valuable insights into the understanding of the essentials of the disease and novel treatment options.

Desmin-related cardiomyopathy (DRCM) is a very rare but severe form of $\operatorname{NICM}(7,8)$. Pathogenic mutations of desmin and $\alpha \mathrm{B}$-crystallin $(\mathrm{CryAB})$ have been identified in familial and sporadic cases of $\operatorname{DRCM}(7,9,10)$. Desmin is an intermediate filament protein that integrates muscle fiber contractility by linking individual myofibrils at the sarcomeric Z-band level (10). CryAB binds to desmin filaments to protect them against stress-induced damage or unfolding (11). Myocardia from DRCM patients exhibit perturbed myofibril alignment and the presence in cardiomyocytes of intracellular aggregates containing desmin and CryAB (9). Although HCM and DRCM have been considered to be separate disease entities, they can both accompany cardiomyocyte hypertrophy, myofibrillar disarray, and accumulation of protein aggregates $(8,12,13)$. 
Because the heart is considered to be under significant physical stress and an organ in which cellular proteins are prone to damage, mechanisms ensuring protein quality control (PQC) are vital to the normal functioning of cardiomyocytes. Such PQC mechanisms include the ubiquitin-proteasome system and macroautophagy (referred to as autophagy unless otherwise specified), alterations of which have been observed in the pathophysiology of cardiac failure in humans and mouse models (14). An important molecule involved in PQC is vacuolar protein sorting 34 (Vps34), the class III phosphoinositide 3-kinase that generates the membrane lipid phosphatidylinositol 3-phosphate $(\mathrm{PI} 3 \mathrm{P})(15,16)$. Vps34 is a critical participant in lipid and protein metabolism by regulating autophagy (17) and the endosomal sorting complexes required for transport-mediated (ESCRT-mediated) protein sorting for degradation (18).

Deletion of Vps34 in mouse muscles results in cardiac hypertrophy and sudden death (19). The mechanism by which loss of Vps34 induces the HCM-like phenotypes remains unknown, although impairment of autophagy may be the basis for the heart abnormalities. To address this issue, we compared heart abnormalities in Mck-Cre; Vps34 $4^{q / f l}\left(\mathrm{cVps} 34^{--}\right)$mice side by side with those in mice lacking Atg5, a master regulator of autophagy ( $\mathrm{Mck}-\mathrm{Cre}$; $\mathrm{Atg} 5^{\text {flfl }}$, hereafter referred to as $c \mathrm{Atg} 5^{--}$). Our aim was to understand the mechanism by which a lack of Vps34 leads to cardiomyopathy: specifically whether a defect in nonautophagic roles of Vps34 is involved. We noted characteristic protein aggregates containing CryAB, an anomaly reminiscent of those observed in cardiomyocytes from patients with myofibrillar cardiomyopathy, in $\mathrm{cVps} 34^{-1-}$ but not in $\mathrm{CAtg}^{-/-}$myocardia. Mechanistically, Vps34 regulates CryAB degradation in a manner dependent on ESCRT-0/Hrs. Our data suggest that the co-occurrence of reduced Vps34 and CryAB ${ }^{+}$aggregates in cardiomyocytes is an alteration that identifies a distinct subset of HCM cases and point to an unexpected common denominator between the pathologies of HCM and DRCM.

\section{Results}

Reduced Vps34 expression in human HCM. To investigate the possible involvement of Vps34 dysregulation in heart diseases, we examined Vps34 protein expression specifically in heart samples from patients with ICM and HCM. Comparative immunostaining of hypertrophied hearts revealed significant reductions in Vps34 protein in cardiomyocytes in 7 out of $18 \mathrm{HCM}$ cases (Figure 1, A and B).

Generation of muscle-specific Vps34-deficient (cVps34-1-) mice. To investigate the molecular mechanism linking decreased expression of Vps34 to HCM, we generated a murine conditional knockout allele $\left(V p s 34^{t}\right)$ encompassing exons 20 and 21 of the $V p s 34 / P i k 3 c 3$ gene, which encode the kinase domain (Supplemental Figure 1A; supplemental material available online with this article; doi:10.1172/jci.insight.89462DS1). Mice harboring $V p s 34^{f}$ were crossed with $m c k$-Cre transgenic mice to delete these $V p s 34$ exons in muscles

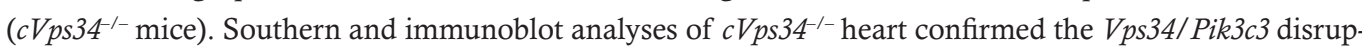
tion and loss of Vps34 protein (Supplemental Figure 1, B and C). Immunostaining revealed punctate Vps34 distribution in $\mathrm{cVps} 34^{+/+}$mouse cardiomyocytes, a pattern not observed in $\mathrm{cVps} 34^{-/-}$hearts (Figure 2A). To visualize PI3P in mouse heart, we crossed $c V p s 34^{-/-}$mice with animals expressing the PI3P reporter EEA1-FYVE-EGFP (20). We detected decreased EGFP-positive $\left(\right.$ EGFP $\left.^{+}\right)$puncta and diffuse distribution of EEA1-FYVE-EGFP in sections of myocardium (Figure 2A), but not liver (Supplemental Figure 1D), of $c$ ps $34^{-1-}$ mice compared with controls. Thus, Vps34 plays a major role in endosomal PI3P production in cardiomyocytes.

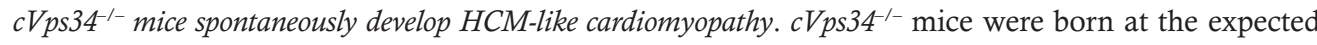
Mendelian ratio, appeared healthy, grew normally, and showed no gross changes in skeletal muscles (Supplemental Figure 2, A-D). However, all $\mathrm{cVps} 34^{-/-}$mutants succumbed to sudden death between postnatal day 80 (P80) and P110 (Figure 2B). Consistent with a previous report (19), the mutant hearts displayed progressive hypertrophy (Figure 2, C and D, and Supplemental Figure 3A). Echocardiography of $c V p s 34^{-1}$ hearts revealed features of HCM, including significant increases in left ventricle mass and left ventricular posterior wall thickness (LVPWd) along with normal left ventricular diastolic diameter (LVDd) (Table 1) (21). However, the mutant hearts also exhibited increased left ventricular end-systolic diameter (LVDs), reduced contractility and ejection fraction (Figure $2 \mathrm{E}$ and Table 1). From these results, we concluded that muscle-specific Vps34 deficiency results in a lethal HCM-like heart disease in mice.

Electrocardiogram by telemetry revealed ventricular arrhythmia and long QT intervals in the mutants (Figure $2 \mathrm{~F}$ and Table 2). The presence of a bundle branch block and increased QRS interval (cVps34 ${ }^{+/+}, 10.8$ $\pm 0.3 \mathrm{~ms}$ vs. $c V p s 34^{--}, 12.5 \pm 0.5 \mathrm{~ms}$ ) in mutant heart indicated a conduction disorder. Histologically, crosssectional diameters of $c V p s 34^{-1-}$ cardiomyocytes were thicker than controls (Supplemental Figure 2B). More- 
A
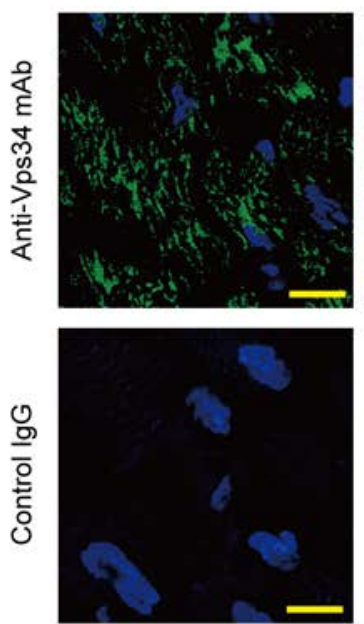

normal
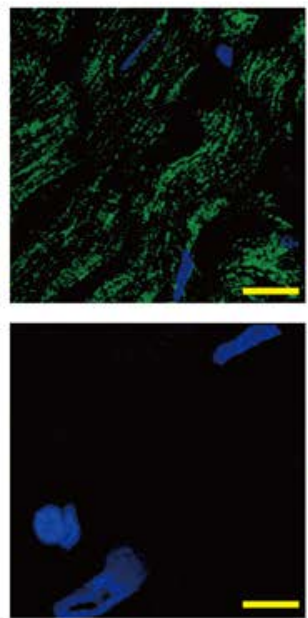

ICM
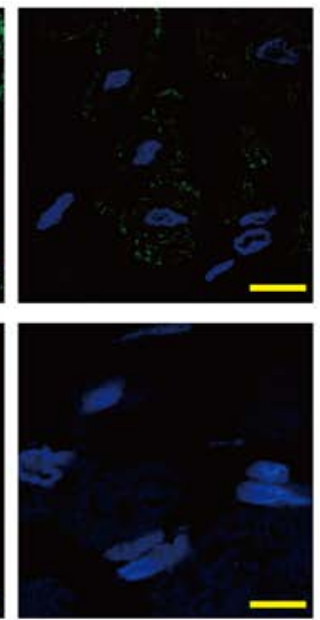

HCM
B

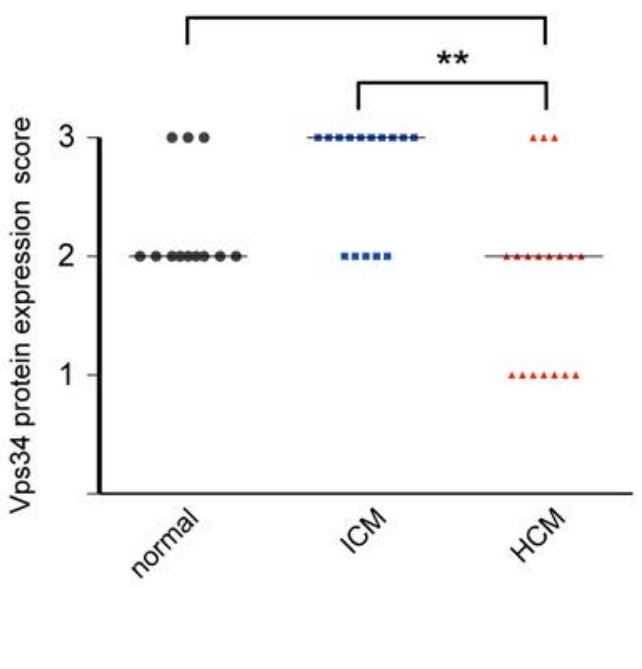

Figure 1. Decreased Vps34 expression in human hypertrophic cardiomyopathy. (A) Representative immunostaining to detect Vps34 protein (green) in cardiac specimens from patients with ischemic cardiomyopathy (ICM, $n=15$ ), hypertrophic cardiomyopathy (HCM, $n=18$ ), or normal individuals ( $n$ = 12). HCM was defined by a wall thickness $\geq 15 \mathrm{~mm}$ in one or more left ventricular myocardial segments according to 2014 ESC guidelines on diagnosis and management of hypertrophic cardiomyopathy. Blue, DAPI staining to detect nuclei. Scale bars: $10 \mu \mathrm{m}$. (B) Quantification of Vps34 protein scores in the specimens in the left panels determined as described in Methods. Bars indicate medians. ${ }^{*} P<0.05$, ${ }^{*} P<0.01$, Kruskal-Wallis test with Dunn's multiple comparisons post-test.

over, isolated cardiomyocytes from $\mathrm{cVps} 34^{-1-}$ hearts also showed increased transverse diameters with normal longitudinal diameters, morphology indicative of pathological hypertrophy (Supplemental Figure 2C).

Protein deposition in Vps34-deficient cardiomyocytes. Gomori trichrome staining revealed intense ectopic signals suggestive of protein deposition in $c V p s 34^{-1-}$ cardiomyocytes (Figure $3 \mathrm{~A}$ ). When left ventricle specimens were compared under the electron microscope, we observed discontinuity of myofibrils, shorter $\mathrm{Z}$ bands and larger intermyofibrillar spaces in $c V p s 34^{-/}$hearts (Figure 3B). Electron-dense depositions, fragmented filaments, amorphous granular structures, and endosomes with electron-dense rims were present in intermyofibrillar spaces of mutant hearts (Figure 3, C-F). Interestingly, these ultrastructural anomalies were similar to those of cardiomyocytes from DRCM patients (22). We found that total protein levels normalized to DNA content were increased in Vps34-deficient hearts (Figure 3G), but that factors promoting protein synthesis, such as mTOR, 4EBP1, and S6, were not upregulated (Figure $3 \mathrm{H}$ ). These findings suggest that a defect in protein degradation might underlie HCM-like cardiomyopathy associated with reduced Vps34.

Autophagic defects in Vps34-deficient cardiomyocytes. Autophagy delivers cytoplasmic proteins and organelles to the lysosome for degradation. Several PI3P-binding proteins contribute to the formation of the omegasomes, from which autophagosomes appear to be generated, and recruit Atg proteins to the isolation membrane $(17,23)$. Atg5 is a master regulator of autophagy and required for lipidation of LC3. The Atg12-Atg5-Atg16L1 complex and the LC3-phosphatidylethanolamine conjugate play important roles in the elongation and closure of the isolation membrane. In light of the abnormal protein deposition and PI3P deficiency in $\mathrm{cVps} 34^{-/-}$hearts, we investigated if autophagy was affected in hearts lacking Vps34 by comparing $c V p s 34^{-/}$cardiomyocytes side by side with autophagy-deficient cardiomyocytes lacking Atg5. Both $c V p s 34^{-/-}$and $m c k C r e-A t g 5^{f l f l}$ (hereafter $\mathrm{cAtg} 5^{-/-}$) cardiomyocytes accumulated p62, a specific substrate for autophagy and an LC3-binding protein (Figure 4A). Furthermore, LC3, which is normally degraded by autophagy, was present at high levels in the hearts lacking Vps34 (Figure 4A and Supplemental Figure 4). Thus, Vps34 is required for normal autophagy in cardiomyocytes.

We then determined the effects of defective autophagy on the heart by examining $c$ Atg $5^{-/-}$mice. Although cardiomyocytes in these mutants contained increased numbers of mitochondria (possibly due to impaired mitophagy) (Supplemental Figure 5), Atg $^{-/-}$mice showed normal heart size, heart/body weight ratio, and heart muscle contractility (Figure 4, B-D), as well as normal LVPWd and LV mass (Supplemental Table 1). Moreover, almost all $\mathrm{cAtg}^{-5^{--}}$mice survived for 150 days (Figure 4E), long after 
Table 1. Hypertrophic cardiomyopathy-like disease in cVps34/- mice

\begin{tabular}{|c|c|c|c|c|}
\hline Parameter & Postnatal day & cVps34 $4^{+/+}$ & cVps34+- & cVps34 \\
\hline \multirow[t]{2}{*}{ LVPWd (mm) } & 20 & $0.61 \pm 0.04$ & $0.53 \pm 0.03$ & $0.56 \pm 0.03$ \\
\hline & 40 & $0.72 \pm 0.03$ & $0.70 \pm 0.05$ & $0.99 \pm 0.07^{A}$ \\
\hline \multirow[t]{3}{*}{ LVDd (mm) } & 20 & $2.81 \pm 0.11$ & $2.76 \pm 0.13$ & $2.79 \pm 0.05$ \\
\hline & 40 & $3.32 \pm 0.10$ & $3.57 \pm 0.10$ & $3.41 \pm 0.10$ \\
\hline & 80 & $3.31 \pm 0.01$ & $3.50 \pm 0.03$ & $3.21 \pm 0.19$ \\
\hline LVDs (mm) & 80 & $1.70 \pm 0.09$ & $1.85 \pm 0.10$ & $2.14 \pm 0.14^{A}$ \\
\hline \multirow[t]{3}{*}{ FS (\%) } & 20 & $52.8 \pm 2.5$ & $50.2 \pm 3.1$ & $48.6 \pm 4.2$ \\
\hline & 40 & $48.8 \pm 2.2$ & $46.2 \pm 2.0$ & $36.3 \pm 1.0^{c}$ \\
\hline & 80 & $47.8 \pm 4.0$ & $42.6 \pm 2.6$ & $31.3 \pm 0.9^{B}$ \\
\hline $\mathrm{EF}(\%)$ & 20 & $82.5 \pm 2.8$ & $78.6 \pm 2.0$ & $72.6 \pm 2.7$ \\
\hline LV mass (mg) & 80 & $88.2 \pm 5.5$ & $110 \pm 7$ & $199 \pm 32^{\mathrm{B}}$ \\
\hline \multirow[t]{3}{*}{ Heart rate (bpm) } & 20 & $447 \pm 24$ & $386 \pm 24$ & $330 \pm 15^{c}$ \\
\hline & 40 & $481 \pm 15$ & $416 \pm 19$ & $372 \pm 17^{A}$ \\
\hline & 80 & $460 \pm 3$ & $482 \pm 19$ & $390 \pm 19^{B}$ \\
\hline
\end{tabular}

Cardiological parameters were determined by echocardiography. LVPWd, left ventricle posterior wall thickness in diastolic phase; LVDd, left ventricle internal dimension in diastolic phase; LVDs, left ventricle internal dimension in end systolic phase; FS, fraction shortening; $E F$, ejection fraction. $c V p s 34^{+/+}$,

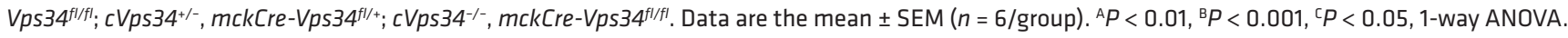

all $c$ Vps $34^{--}$mice had died from heart failure (Figure 2B). Thus, the severe cardiac abnormalities and sudden death of $c V p s 34^{-1-}$ mice are not solely attributable to impaired autophagy.

Vps34 deficiency induces accumulation of K63-linked polyubiquitinated proteins. Because Vps34 deletion resulted in greater protein deposition and more severe functional defects in the heart than $\operatorname{did} A \operatorname{tg} 5$ deletion, we sought to identify a protein degradation system that depends on Vps34 but not on Atg5, and whose disruption might trigger the HCM-like disease. Polyubiquitination marks unfolded or misfolded proteins for degradation, and proteins linked to ubiquitin through $\mathrm{K} 63(\mathrm{~K} 63 \mathrm{pUb})$ are targeted for proteolysis via the multivesicular body (MVB) pathway $(24,25)$. In the first step of MVB formation, the ubiquitinated cargo is sequestered by the ESCRT-0 complex, a key subunit of which is the Hrs protein that harbors 2 ubiquitinbinding domains and a PI3P-binding FYVE domain (18).

We transfected P19.CL6 cells with siRNA blocking Vps34 expression (Supplemental Figure 6A) and detected an abnormal accumulation of $\mathrm{K} 63 \mathrm{pUb}$ proteins (Figure 5A, left). Hrs-immunoreactive puncta were decreased in Vps34-depleted P19.CL6 cells (Figure 5A). This reduction was most likely due to cytoplasmic dispersion of Hrs caused by insufficient endosomal PI3P (Figure 2A), since Hrs levels were normal in whole lysates of Vps34-depleted P19.CL6 cells but reduced in the membrane fraction (Supplemental Figure 6B). Immunoblotting of $c V p s 34^{--}$and $c A t g 5^{-1-}$ hearts revealed that $\mathrm{K} 63 \mathrm{pUb}$ proteins accumulated only in the former (Figure 5B). This accumulation commenced at P20, when cardiac functions are still normal in $\mathrm{cVps}^{-4^{--}}$mice, and continued to high levels as the mutants aged. On the other hand, the levels of K48-linked polyubiquitinated proteins were higher in both Vps34 KO and Atg5 KO compared with their controls, and there were no differences in the accumulation between the 2 genotypes. In $\mathrm{cVps} 34^{-1}$ cardiomyocytes, $\mathrm{K} 63 \mathrm{pUb}$ proteins formed aggregates of ring-like structures in the cytoplasm, and most of these aggregates were positive for p62 (Figure 5C). Collectively, these data suggest that a defect in the Hrsdependent MVB pathway that leads to $\mathrm{K} 63 \mathrm{pUb}$ protein accumulation in cardiomyocytes may underlie the heart pathogenesis associated with Vps34 deficiency.

Lack of Vps34 leads to accumulation of CryAB. Next, we sought to identify a specific protein(s) that underwent polyubiquitination through K63-linkage and accumulated in Vps34-deficient hearts. We previously 
A

cVps $34^{+/+}$

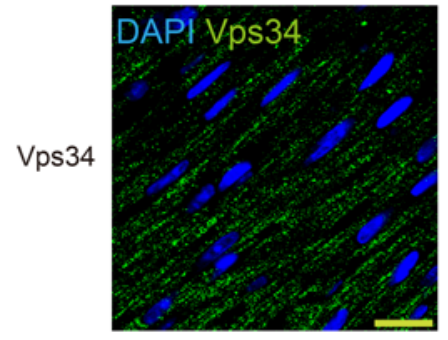

cVps34 $4^{+/+}$

EGFP-EEA1-FYVE Tg

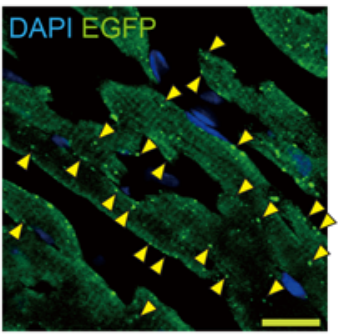

cVps34/-

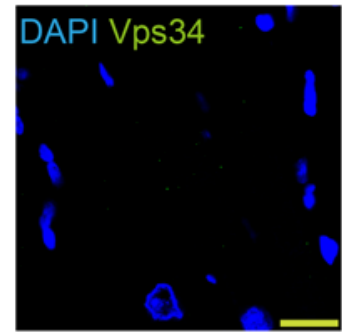

cVps34 $4^{-/-}$

EGFP-EEA1-FYVE Tg
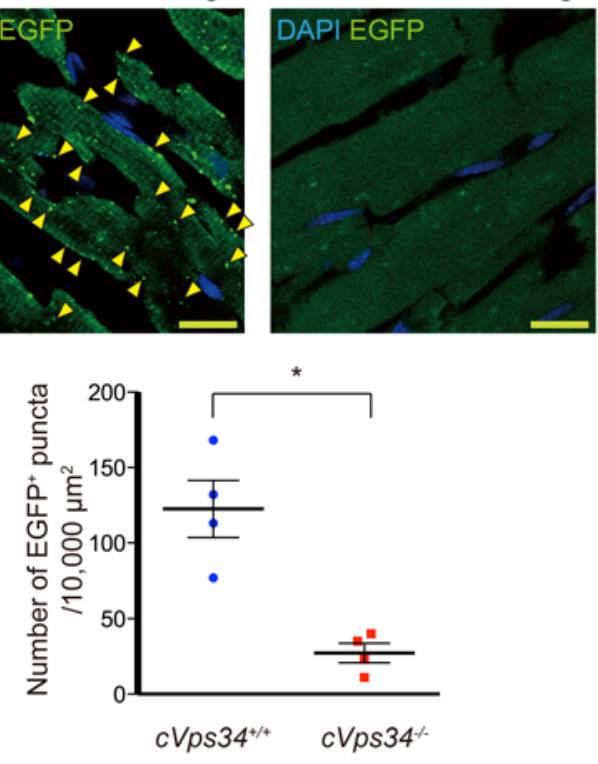

B

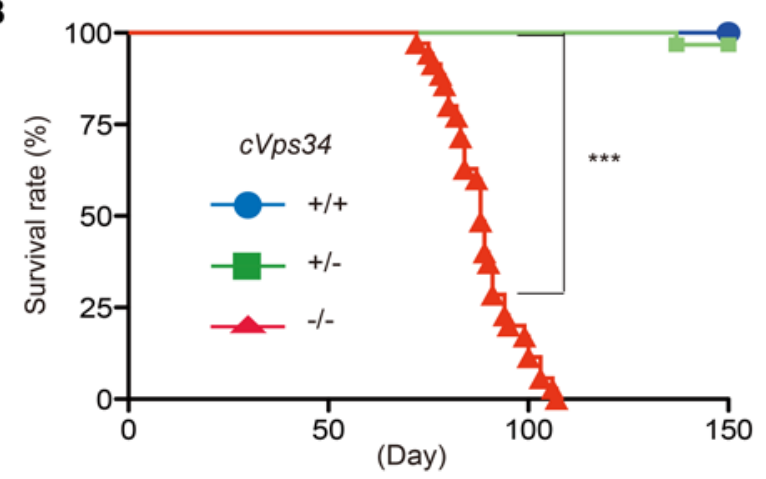

C

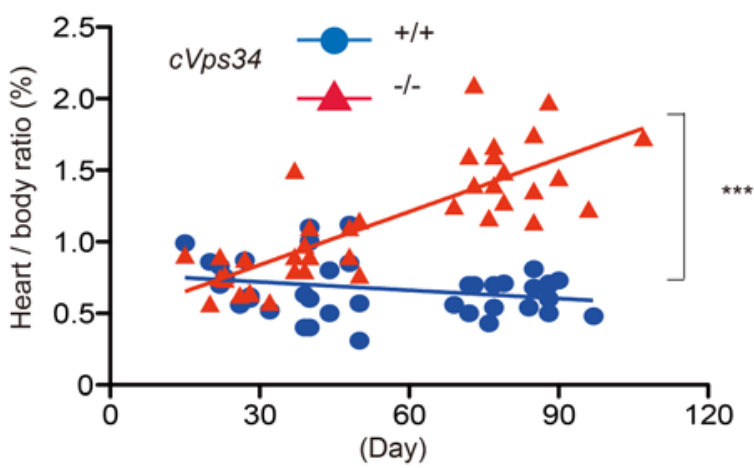

D

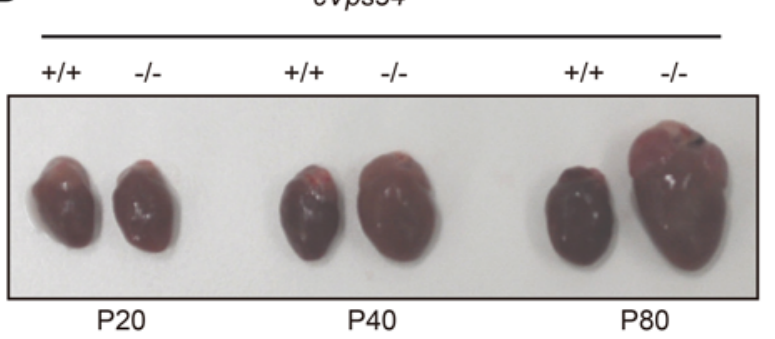

E

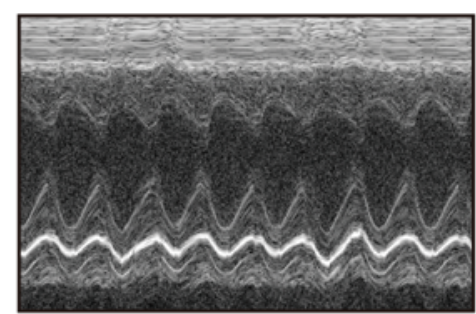

cVps34/-

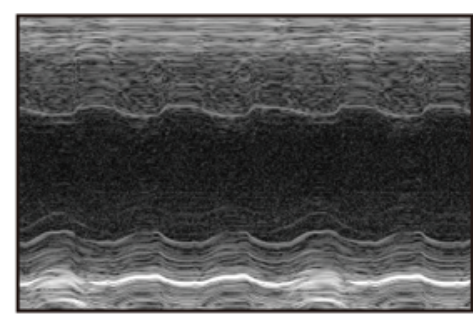

$\mathbf{F}$
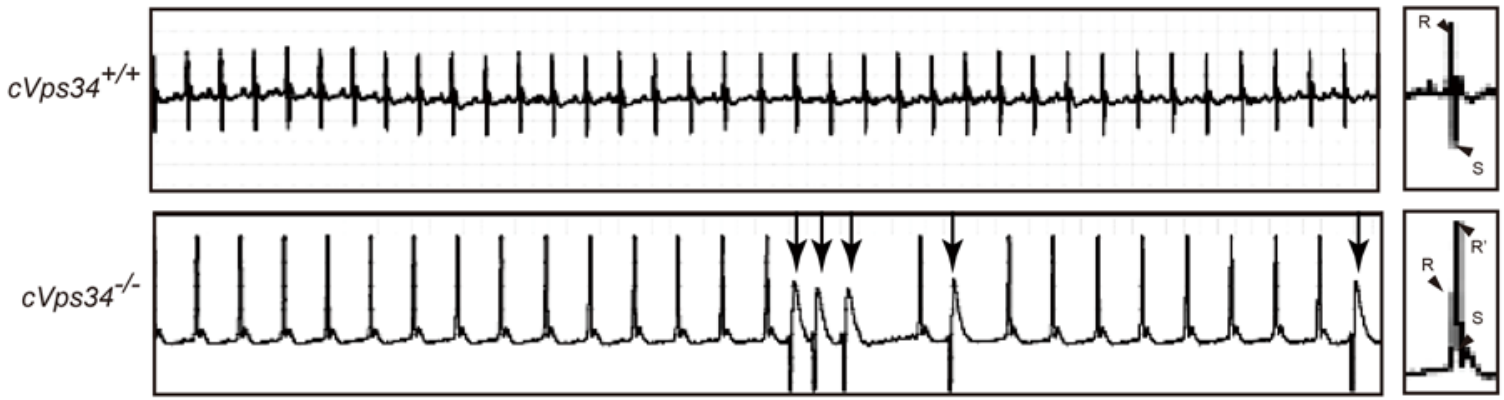

Figure 2. Vps34-deficient mice develop hypertrophic cardiomyopathy-like cardiomyopathy. (A) Immunofluorescence analyses to detect (upper panels) Vps34 (green) and (middle panels) a phosphatidylinositol 3-phosphate bioprobe (CFP-EEA1-FYVE) in cardiac left ventricular sections from mice of the indicated genotypes. Representative images are shown. Blue, DAPI staining to detect nuclei. Arrowheads indicate GFP+ puncta. Scale bars: $10 \mu \mathrm{m}$. (Lower panels) GFP' puncta were counted in 4 randomly chosen fields $\left(10,000 \mu \mathrm{m}^{2}\right)$ for each sample $(n=4 /$ group $) .{ }^{*} P<0.01,2$-tailed Student's $t$ test. (B) 


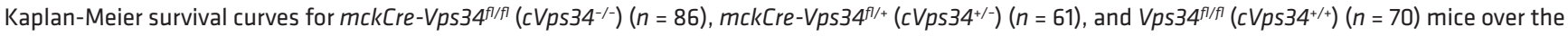
indicated period. All cVps34 ${ }^{-/-}$mice were dead by P110. ${ }^{* *} P<0.001$, log-rank test. (C) Heart weight to body weight ratios for individual $c V p s 34^{+/+}(n=40)$ and $c$ ps $34^{-/-}(n=39)$ mice over the indicated period. Linear regression lines are shown. ${ }^{* *} P<0.001$, Pearson's test. (D) Macroscopic views of hearts from $\mathrm{cVps}_{3} 4^{+/+}$and $\mathrm{cVps34} 4^{-/-}$mice at the indicated ages. Results are representative of at least 10 hearts examined per group. P, postnatal day. (E) Representative M-mode echocardiograms of $c$ ps $34^{+/+}$and $c V p s 34^{-/-}$mice $(n=6 / g r o u p)$ at P80. (F) Electrocardiograms at P80 showing short runs of premature ventricular contraction (arrows), QT elongation, and bundle branch block (right panels) in a cVps34

reported that lens-specific deletion of Vps34 causes congenital cataracts in mice (26). Similarly, autosomal dominant missense mutations in the human $C r y A B$ gene result in both cataracts and cardiomyopathy (9, 27). These findings prompted us to explore CryAB's role in the $\mathrm{cVps} 34^{-/-}$phenotype.

Western blot analysis of CryAB in an NP40-insoluble fraction prepared from the heart revealed that CryAB accumulated in $c V p s 34^{-1-}$ hearts starting at P20 (Figure 6A), suggesting that Vps34 controls CryAB degradation in cardiomyocytes. We then subjected $c V p s 34^{-/-}$heart lysates to immunoprecipitation with anti-CryAB antibody $(\mathrm{Ab})$ followed by immunoblotting with anti-K63pUb Abs and viceversa to determine whether CryAB is among the $\mathrm{K} 63 \mathrm{pUb}$ proteins that accumulate in the absence of Vps34. We consistently detected a band of $\sim 40 \mathrm{kDa}$, the size expected for CryAB protein modified with di- or tri-ubiquitin (Figure 6, B and C). We next cotransfected Vps34-depleted P19.CL6 cells with Myc-tagged CryAB plus FLAG-tagged WT, K48R-, or K63R-ubiquitin. K48R and K63R are mutations that prevent K48- and K63-linked polyubiquitination, respectively. A CryAB band of $\sim 40 \mathrm{kDa}$ was readily detected in anti-FLAG immunoprecipitates prepared from cells expressing WT or K48R ubiquitin, but not from cells expressing K63R ubiquitin (Figure 6D). Thus, CryAB is ubiquitinated via K63 in the absence of Vps34.

CryAB is a causative gene mutated in $\operatorname{DRCM}(7,9)$. Because intra-sarcoplasmic aggregates containing CryAB and desmin are a DRCM hallmark, we immunostained the aggregates in our $\mathrm{cVps} 34^{-1-}$ mouse hearts to determine their contents. In WT cardiomyocytes, CryAB localized with desmin in Z bands and at the plasma membrane (Figure $6 \mathrm{E}$ ), consistent with a previous report (28). In contrast, the coordinated network of myofibrils observed in WT heart was disrupted in Vps34-deficient hearts (Figure 6E). Notably, $c V p s 34^{-1-}$ cardiomyocytes exhibited $\mathrm{CryAB}^{+} / \mathrm{desmin}^{+}$aggregates and vacuolar structures, recapitulating DRCM pathology. In addition to $\mathrm{CryAB}^{+}$and desmin ${ }^{+}$, p62 was colocalized at the periphery of the vacuolar structures in the absence of $\mathrm{Vps} 34$, echoing a study of ventricular myocytes overexpressing the pathogenic CryAB mutant protein (29). Thus, without Vps34, pathogenic protein aggregation occurs that can precipitate a disease which can be diagnosed with HCM-like cardiomyopathy but exhibits histological abnormalities characteristic of DRCM.

CryAB undergoes degradation in an ESCRT-0-dependent manner. Hrs and signal transducing adaptor molecule (STAM) proteins comprise the ESCRT-0 complex. We found that CryAB was increased in P19.CL6 cells upon siRNA-mediated depletion of Hrs or STAM1 plus STAM2, implying that K63-linked polyubiquitinated CryAB is recognized by ESCRT-0 at endosomal membranes (Figure 7 and Supplemental Figure 6). Without Vps34, there is likely insufficient PI3P in endosomal membranes to facilitate Hrs binding to $\mathrm{K} 63 \mathrm{pUb}$ proteins, including CryAB. ESCRT-mediated proteolysis may then be impaired, leading to cytoplasmic CryAB accumulation. K63pUb proteins other than CryAB may also be involved in the heart abnormalities in $\mathrm{cVps} 34^{-/-}$mice.

Human HCM hearts showing decreased expression of Vps34 exhibit deposits containing CryAB, desmin, p62, and K63pUb proteins. The above data suggest that impaired ESCRT-mediated degradation of K63pUb proteins (including $\mathrm{Cry} \mathrm{AB}$ ) is the mechanism by which a lack of Vps34 leads to the HCM-like cardiomyopathy in mice. To address whether this proposed molecular mechanism also holds true for human HCM, we reexamined our original 18 human HCM myocardial samples (Figure 1). Immunostaining to detect CryAB, desmin, and p62 in these samples revealed accumulations of $\mathrm{CryAB}^{+} / \mathrm{desmin}^{+}$aggregates in 4 of these cases (Figure 8A, Table 3, and Supplemental Figure 7). Significantly, all 4 of these HCM samples had also lost Vps34 protein and displayed strong positivity for $\mathrm{p} 62$ and K63pUb proteins (Figure 8B, Table 3, and Supplemental Figure 8), replicating our results in $c V p s 4^{-/-}$mouse hearts. These Cry$\mathrm{AB}^{+} /$desmin $^{+} / \mathrm{p} 62^{+} / \mathrm{K} 63 \mathrm{pUb}^{+}$protein deposits were consistently absent in normal or ICM human heart tissues (Supplemental Tables 2 and 3). Thus, the co-occurrence of reduced Vps34 and $\mathrm{CryAB}^{+} / \mathrm{desmin}^{+} /$ $\mathrm{p}^{6} 2^{+} / \mathrm{K} 63 \mathrm{pUb}^{+}$aggregates in cardiomyocytes is a signature alteration that may identify a distinct subset of HCM cases in both humans and mice. 
Table 2. cVps34-/- mice show abnormal electrocardiographic parameters

\begin{tabular}{lcc}
\hline Parameter & Vps34+/+ & \multicolumn{1}{c}{ Vps34 $^{-/-}$} \\
RR Interval (sec) & $0.112 \pm 0.002$ & $0.114 \pm 0.002$ \\
Heart Rate (bpm) & $540 \pm 11$ & $528 \pm 10$ \\
PR Interval (sec) & $0.036 \pm 0.001$ & $0.037 \pm 0.002$ \\
P Duration (sec) & $0.014 \pm 0.001$ & $0.017 \pm 0.003$ \\
QRS Interval (sec) & $0.011 \pm 0$ & $0.013 \pm 0.001^{\mathrm{A}}$ \\
QT Interval (sec) & $0.046 \pm 0.002$ & $0.061 \pm 0.004^{\mathrm{B}}$ \\
QTc (sec) & $0.043 \pm 0.001$ & $0.057 \pm 0.003^{\mathrm{B}}$ \\
JT Interval (sec) & $0.035 \pm 0.001$ & $0.049 \pm 0.004^{\mathrm{A}}$
\end{tabular}

Male $c$ vps34/- mice $(n=6)$ and littermate control cVps34+/+ mice $(n=6)$ were analyzed at P80 for the indicated parameters. Data are the mean \pm SEM. ${ }^{A} P<0.05,{ }^{\mathrm{B}} P<0.01,2$-tailed Student's $t$ test.

\section{Discussion}

This study has uncovered a possible causal linkage between human HCM and altered cardiac expression of the lipid kinase Vps34. One of the strengths of our study is the consistency between the findings in a subset of HCM patients and the Vps34-deficient mouse model. $c V p s 34^{-1-}$ mice suffered from HCM-like abnormalities that culminated in sudden death. Notably, hearts of affected mice displayed histological anomalies, particularly accumulation of $\mathrm{CryAB}^{+} /$des$\mathrm{min}^{+} / \mathrm{p} 62^{+} / \mathrm{K} 63 \mathrm{pUb}^{+}$aggregates that are considered typical of DRCM. Equivalent protein aggregates were found in cardiomyocytes from HCM patients exhibiting reduced Vps34 expression. It is, therefore, likely that impaired proteostasis of CryAB is a key to the cardiac manifestations of HCM as it is for DRCM.

Phosphorylation of mTOR and S6 kinase was downregulated at P40 and P80 in $\mathrm{cVps} 34^{-1-}$ hearts (Figure $3 \mathrm{H}$ ), but rapamycin treatment did not promote accumulation of K63pUb proteins or CryAB in P19.CL6 cells (data not shown). Given that inhibition of mTORC1 was shown to reduce pathological hypertrophy and heart failure in mice with cardiomyopathies (30), the observed downregulation of mTOR phosphorylation in $\mathrm{cVps} 34^{-/-}$hearts may be a compensatory response to the decreased activity of protein degradation machinery.

Several studies have shown that the 2 major protein degradation machineries, namely autophagy and the proteasome system, are involved in protein quality control in cardiomyocytes $(31,32)$. Our data have revealed that the ESCRT-dependent MVB pathway, which is responsible for degrading K63pUb proteins (25) also may play a pivotal role in cardiac proteinopathies. Specifically, the Vps34-ESCRT-0 axis plays a key role in regulating proteostasis of CryAB. Although the canonical cargos of the MVB pathway are membrane proteins, recent studies have proposed a new type of autophagy called endosomal microautophagy, by which soluble cytosolic proteins are incorporated into intraluminal vesicles of MVBs in ESCRTdependent manners (33-35). ESCRT-dependent degradation of the nontransmembrane protein CryAB revealed in this study demonstrates the physiological relevance of endosomal microautophagy at a wholeanimal level. Alterations to the MVB pathway have been linked to human disease in other contexts. For example, mutations in the ESCRT-III subunit Vps2/CHMP2B are associated with frontotemporal dementia, and with amyotrophic lateral sclerosis characterized by accumulation of $\mathrm{pUb}^{+}$protein aggregates in affected neurons $(36,37)$.

Our side-by-side comparison of $c V p s 34^{-/-}$and $c A t g 5^{-/-}$mice showed that impaired autophagy on its own causes only modest cardiac abnormalities, if any. We propose that the MVB pathway and autophagy are protein degradation systems that can cooperate or compensate for one another, and that severe cardiomyopathy occurs when both mechanisms are simultaneously compromised. Figure 9 depicts a model of how Vps34 might maintain cardiomyocyte integrity. Under physiological conditions (and thus normal Vps34 expression), denatured or misfolded CryAB becomes ubiquitinated via K63 linkage and is destined for degradation through the MVB pathway/endosomal microautophagy; no CryAB accumulates in the cytoplasm. When Vps34 expression is decreased or absent, CryAB that is denatured and then ubiquitinated cannot undergo normal cargo sorting via the MVB pathway and so accumulates in the cytoplasm. This CryAB then binds to $\mathrm{p} 62$, which has a preference for binding to K63-pUb proteins and functions as a cargo adaptor that targets ubiquitinated proteins for autophagy (38). However, because autophagy is also impaired in the absence of Vps34, these CryAB-p62 complexes cannot be degraded and form aggregates. In contrast with the function of native CryAB, which binds to desmin and prevents it from unfolding, the ubiquitinated CryAB and/or abnormal CryAB-p62 complexes that arise in the absence of Vps34 might interfere with the chaperone-like activity of native CryAB, as has been shown for CryAB ${ }^{\mathrm{R} 120 \mathrm{G}}$ protein $(27,39,40)$. Under such conditions, unfolded desmin would predominate over native desmin in cardiomyocytes, leading to the accumulation of aberrant ternary complexes of CryAB, p62, and desmin. This impaired homeostasis would then result in destruction of myofibrils and subsequently HCM and heart failure. We speculate that this mechanism may explain why Vps34 disruption in mice culminates in HCM-like cardiomyopathy showing histopathological features similar to those of DRCM.

HCM and DRCM are causally related to mutations in genes encoding proteins localized at the sarcomere (e.g., MYH7, MYBPC3) and Z band (e.g., CryAB, desmin), respectively. These proteins are all essential for myofibrillar structure and function. Myocardia that were derived from patients clinically diagnosed 
A

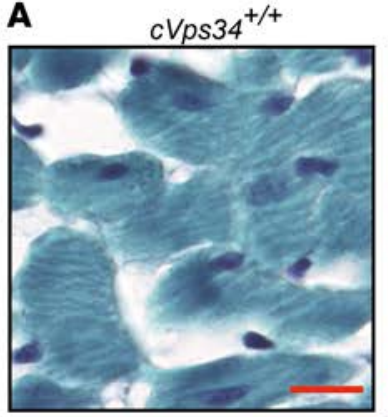

C

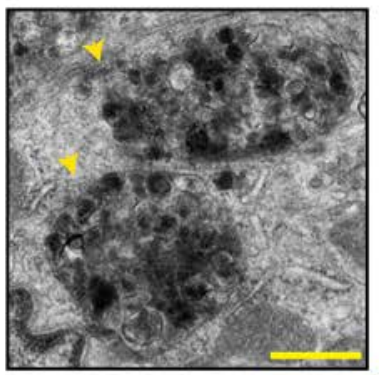

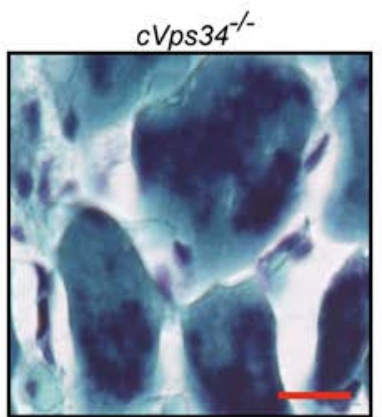

D

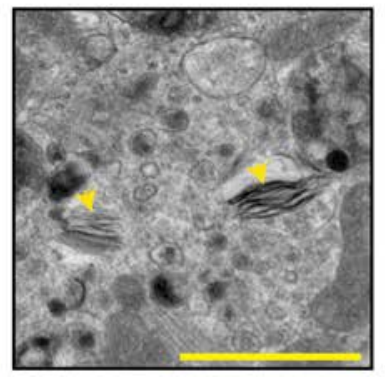

$\mathbf{F}$
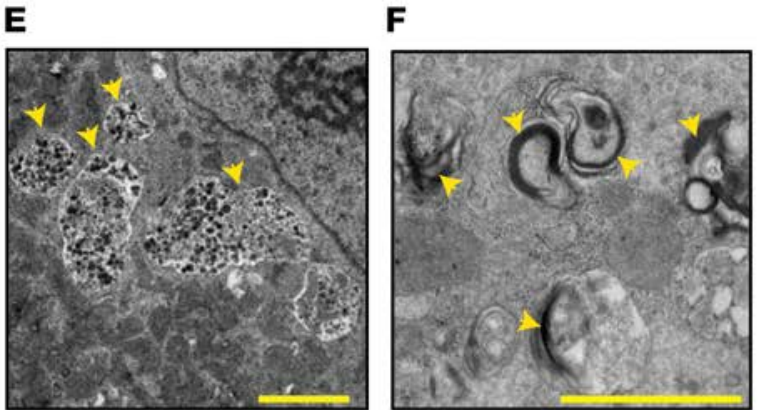

G

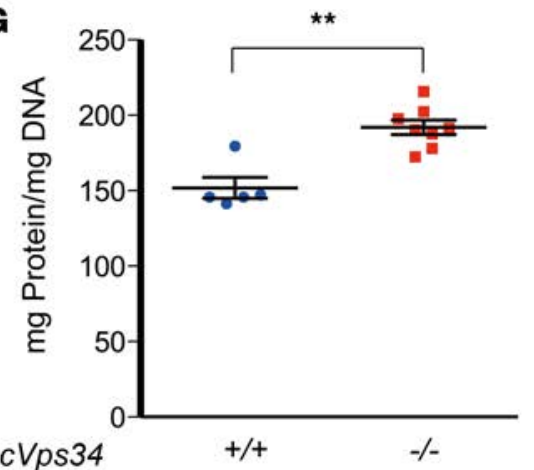

B
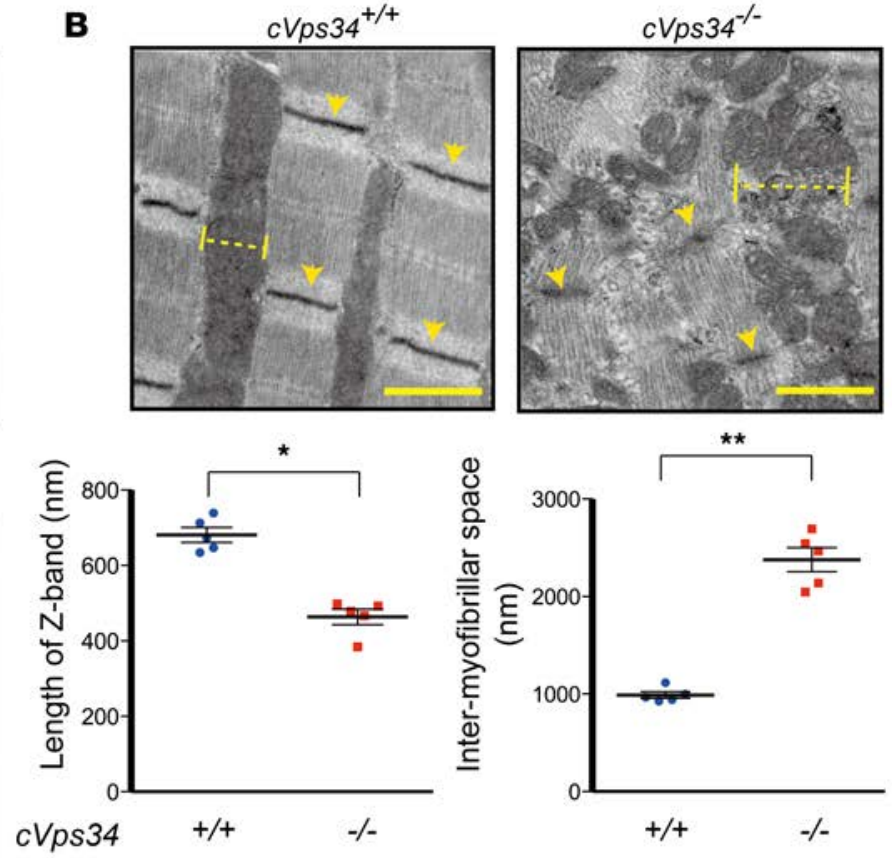

H

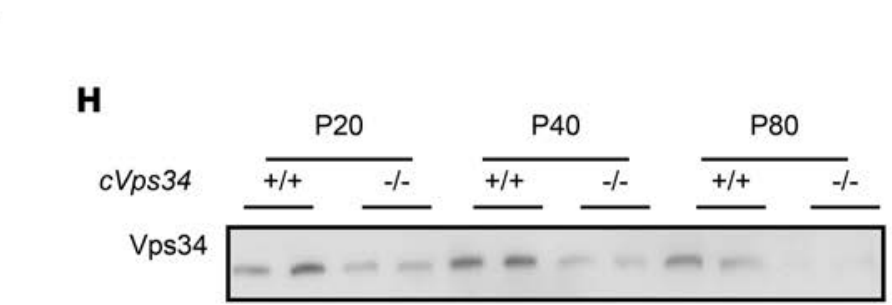

Phospho-mTor

(S2448)

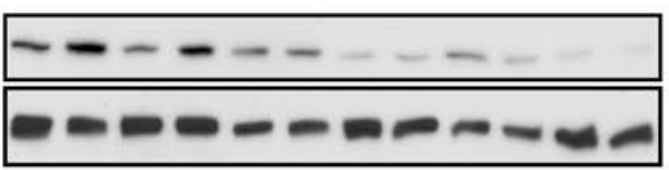

Phospho-4EBP1

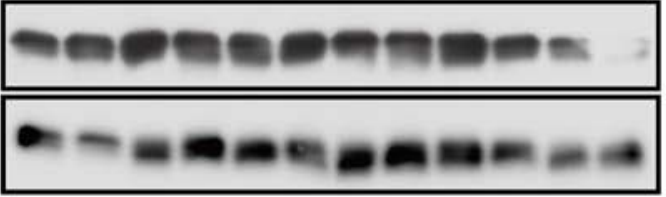

Phospho-S6

(S235/S236)

S6

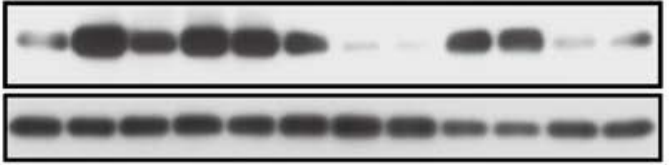

GAPDH

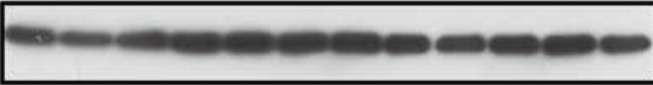

Figure 3. Myofibrillar disorganization accompanied by protein accumulation in Vps34-deficient murine hearts. (A) Representative Gomori trichromestained sections of left ventricles from mckCre-Vps34 f//fl$\left(c V p s 34^{-/-}\right)$and $V p s 34^{f / / f}\left(c V p s 34^{+/+}\right)$mice at postnatal day (P) 80 ( $n=4 /$ group). Aberrant protein deposits appear as metachromasia (amorphous and dark-colored areas) in cVps34-- myocardia. Scale bars: $100 \mu \mathrm{m}$. (B) Upper panels: Electron micrographs of $\mathrm{cVps} 34^{+/+}$and $\mathrm{cVps34^{-/- }}$ cardiomyocytes. $Z$ bands (arrowheads) and intermyofibrillar spaces (dotted lines) were perturbed in the mutant. Scale bars: $1 \mu \mathrm{m}$. Lower panels: Quantification of lengths of Z-bands and intermyofibrillar spaces ( $n>10$ cells examined/mouse, $n=5 / \mathrm{group})$. Data are the mean \pm SEM. (C-F) Electron micrographs showing abnormal ultrastructure in cVps34-/- cardiomyocytes ( $n>100$ cells examined $/ \mathrm{mouse}, n=3-6$ examined/group) at P80. (C) Electron-dense depositions, (D) fragmented filaments (indicating Z-band disruption), (E) amorphous granular structures present in close proximity to cardiomyocyte nuclei, and (F) endosomes with electron-dense rims. Scale bars: $1 \mu \mathrm{m}$. (C) Total protein levels in $c V p s 34^{+/+}$and $c V p s 34^{-/-}$hearts $(n=5-8$ /group). Data are the mean \pm SEM of values normalized to DNA content. (H) Immunoblot to detect the phosphorylation of the indicated signaling molecules involved in protein synthesis in heart extracts from $c V p s 34^{+/+}$and $c V p s 34^{-/-}$mice of the indicated ages. GAPDH, loading control. Results are representative of at least 3 independent trials. ${ }^{*} P<0.05,{ }^{* *} P<0.01,2$-tailed Student's $t$ test. 
Table 3.

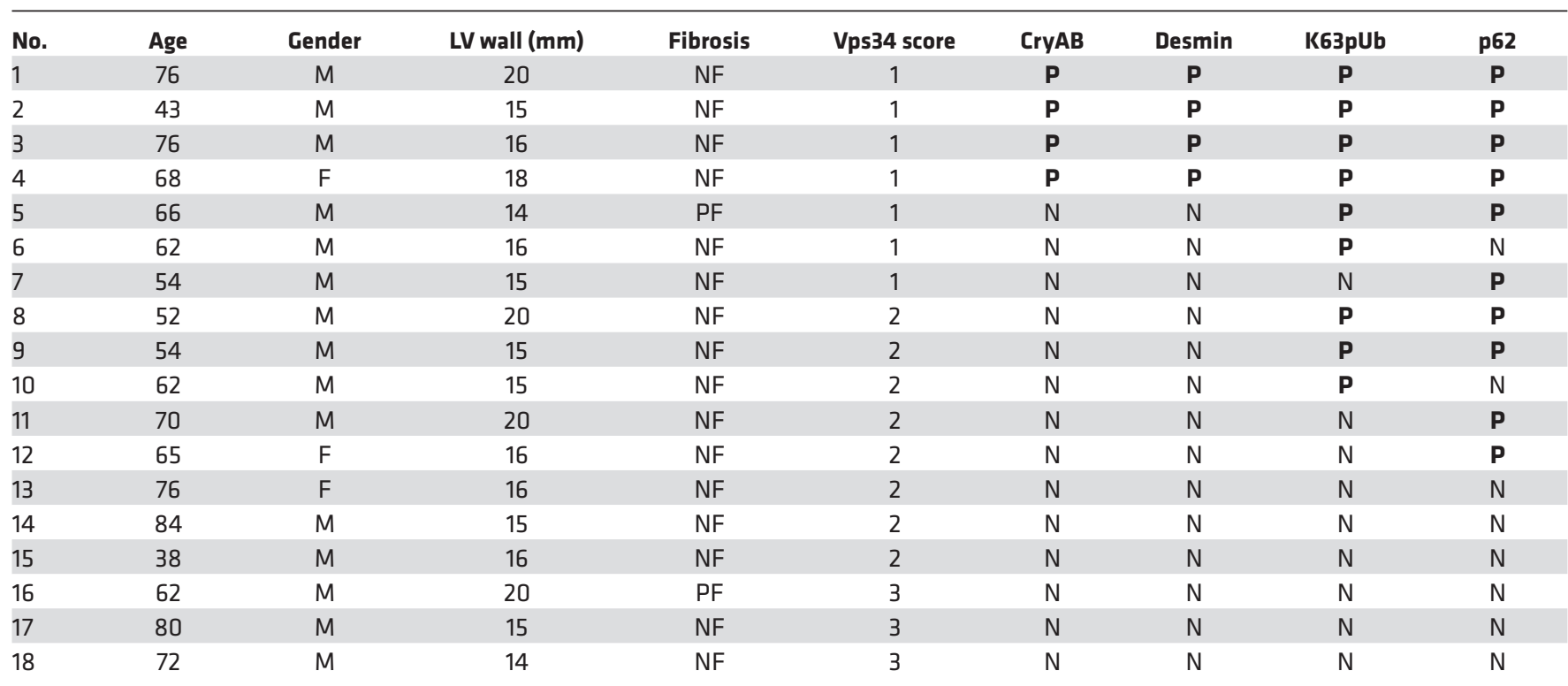

Characteristics of the hypertrophic cardiomyopathy (HCM) patients whose myocardial samples were analyzed by IHC. Vps34 expression levels were scored as in Supplemental Figure 9. PF, presence of myocardial fibrosis within left ventricle (LV). NF, absence of myocardial fibrosis. N, normal localization of CryAB or desmin, or no detectable signal for K63pUb or p62. P, presence of abnormal aggregates containing the indicated proteins. All patients were diagnosed with HCM according to 2014 European Society for Cardiology Guidelines of diagnosis and management of hypertrophic cardiomyopathy.

with HCM and that also displayed decreased Vps34 were found to accumulate $\mathrm{CryAB}^{+} / \mathrm{desmin}^{+}$aggregates (Figure 8, A and B). We propose a model of these 2 cardiomyopathies in which their overlapping manifestations are due in some way to Vps34 dysregulation. Such a causative or modulating mechanism might account for the considerable clinical heterogeneity of miscellaneous symptoms in HCM and DRCM patients, even among those with the same disease-causing mutation (3-5). In summary, our study offers fresh insights into the pathogenesis of HCM. The potentially novel molecular mechanism proposed here may advance the diagnosis of cardiac disorders and assist in the development of new therapies.

\section{Methods}

Human myocardial specimens. Paraffin-embedded human cardiac tissue arrays annotated with clinical information (Tissue Microarray Human Cardiovascular Tissue: Myocardial Hypertrophy and Tissue Microarray Human Cardiovascular Tissue: Myocardial infarction) were purchased from Provitro. These arrays contained samples from heart disease patients with cardiac hypertrophy arising from various etiologies, including HCM $(n=18)$ and ICM $(n=15)$. Sixteen of $18 \mathrm{HCM}$ cases fulfilled the diagnostic criteria in the 2014 ESC Guidelines on diagnosis and management of hypertrophic cardiomyopathy (2) and 2 of those had lesser degrees of left ventricular wall thickening $(13-14 \mathrm{~mm})$ and were histologically positive for hypertrophy of cardiomyocytes. Normal cardiac tissue samples $(n=12)$ consisted of 7 autopsied cases that were determined to be free of heart diseases and were obtained from our institution, and 5 cases from a second human cardiac tissue array (Tissue Microarray Human Cardiovascular Tissue: Myocardial Infarction [Provitro]). Hearts of autopsy cases were fixed with $10 \%$ formaldehyde and embedded in paraffin. For all control samples, myocardial tissue from the left ventricular free wall was analyzed and found to lack any significant histological changes.

Protein determinations in human myocardial specimens. For Vps34, the above myocardial tissue arrays and autopsy specimens were immunostained with anti-Vps34 Ab (clone EPR5301). Staining intensity of each cell was arbitrarily scored as being at level $0,+1$, or $+2(41)$. This Vps34 protein level was multiplied by the percentage of immunoreactive cells among total cells counted to generate 3 groups: group 1, Vps34 protein expression score of less than 50 (low Vps34 protein); group 2, score 50-150 (medium); and group 3 , score greater than 150 (high). For each tissue section, anti-Vps34 staining intensity was determined for 
A

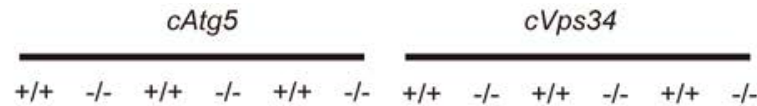

p62
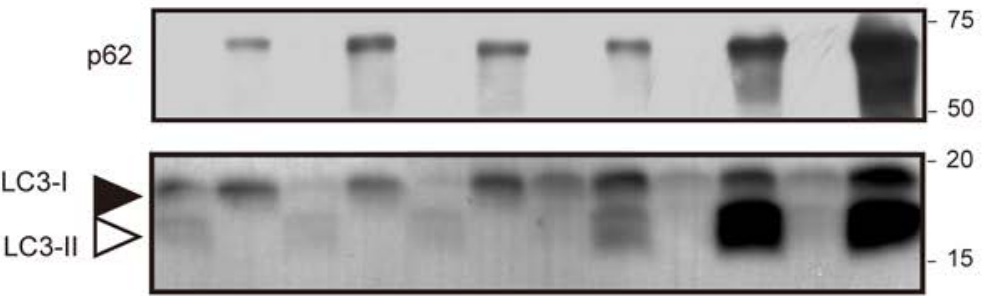

Atg5

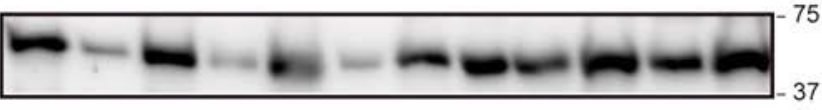

Vps34

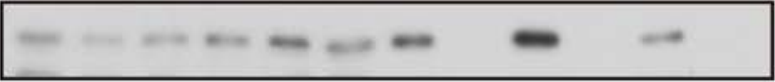

GAPDH

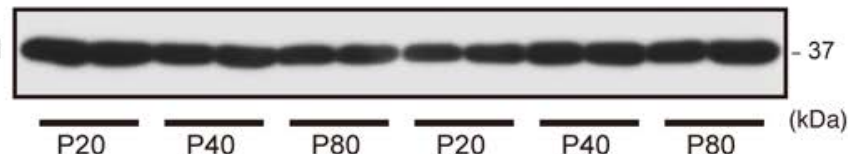

D
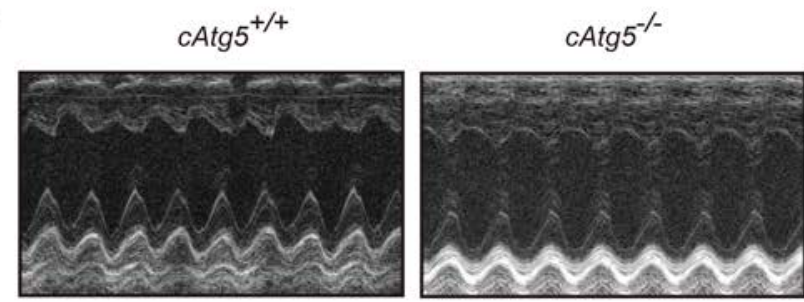

B
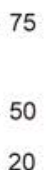

15

75

100

37

(kDa)

\section{E}

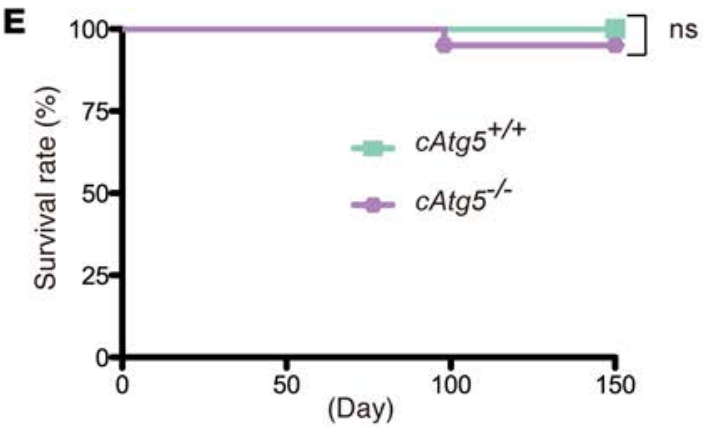

Figure 4. Vps34 is required for autophagy-mediated protein degradation in cardiomyocytes. (A) Immunoblot of heart lysates from Atg $5^{f / / f l}\left(\mathrm{cAtg} 5^{+/+}\right)$, mckCre-Atg $5^{f / f l}\left(\mathrm{CAtg}^{-/-}\right), c \mathrm{cVs} 34^{+/+}$, and $\mathrm{cVps} 34^{-/-}$hearts to detect the indicated proteins. Results are representative of 3 trials. (B) Representative macroscopic views of hearts from $c \operatorname{Atg} 5^{+/+}(n=17)$ and $c \operatorname{Atg} 5^{-/-}(n=18)$ mice at the indicated ages. P, postnatal day. (C) Heart/body weight ratios for $c A t g 5^{+/+}$ $(n=17)$ and $c A t g 5^{-1-}(n=18)$ mice. ns, not significant. (D) Representative M-mode echocardiograms in $c A t g 5^{+/+}$and $c A t g 5^{-/-}$mice at P80. (E) Kaplan-Meier survival curves for $\mathrm{CAtg}^{+/+}(n=80)$ and $\operatorname{cAtg5^{-/-}}(n=88)$ littermate mice.

more than 100 cells in at least 3 distinct areas of the slide. This method allowed classification of $80 \%$ of control samples in group 2 of Vps34 protein expression. Representative images for each group are shown in Supplemental Figure 9. For other proteins, immunostaining was performed using the appropriate Abs (see Antibodies section in Methods). No control heart specimens exhibited abnormal aggregates of K63pUb, p62, desmin, or CryAB proteins.

Mice. The $V p s 4^{f}$ allele was generated using standard homologous recombination in E14K embryonic stem (ES) cells. An EcoRI-ApaI genomic fragment containing exons 20 and 21 of the murine Vps34/Pik3c3 gene, plus the $P G K-H y g r o^{r}-p A$ cassette, were flanked by 3 loxP sequences. The diphtheria toxin A (DTA) gene was inserted downstream of the short arm to allow negative selection against random integration of the vector. The linearized construct was electroporated into $1 \times 10^{7} \mathrm{E} 14 \mathrm{~K}$ mouse ES cells. ES cell colonies resistant to G418 $(0.3 \mathrm{mg} / \mathrm{ml})$ were screened by PCR. Recombinant colonies were confirmed by hybridizing a Southern blot of PstI-digested genomic DNA to a 336-bp 5' flanking probe: forward primer, 5'-CGCATTCTAGAGCATAACATA-3'; reverse primer, 5'-ACGTCATTACCTGAATGCTTC-3'. Targeted ES cells were injected into C57BL/6 blastocysts. Chimeric male mice were crossed with C57BL/6 females to obtain $V p s 34^{\theta /+}$ mice. The following primer set was used to detect the WT $V p s 34$ and $V p s 34^{A}$ alleles: forward primer, 5'-CGGCACCTGGATAACCTTTTG-3'; reverse primer, 5'-TTCCCCTAAGTGTCCTTCCTGTG-3'. For muscle-specific Vps34-deficient mice generated by crossing with mck-Cre 
A
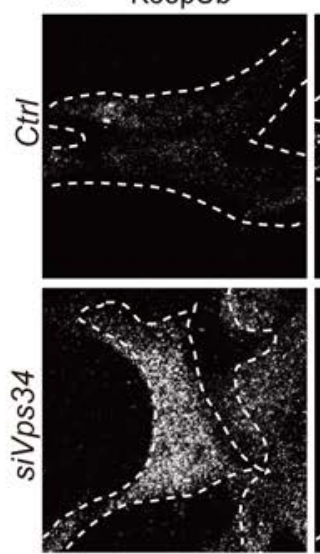

C
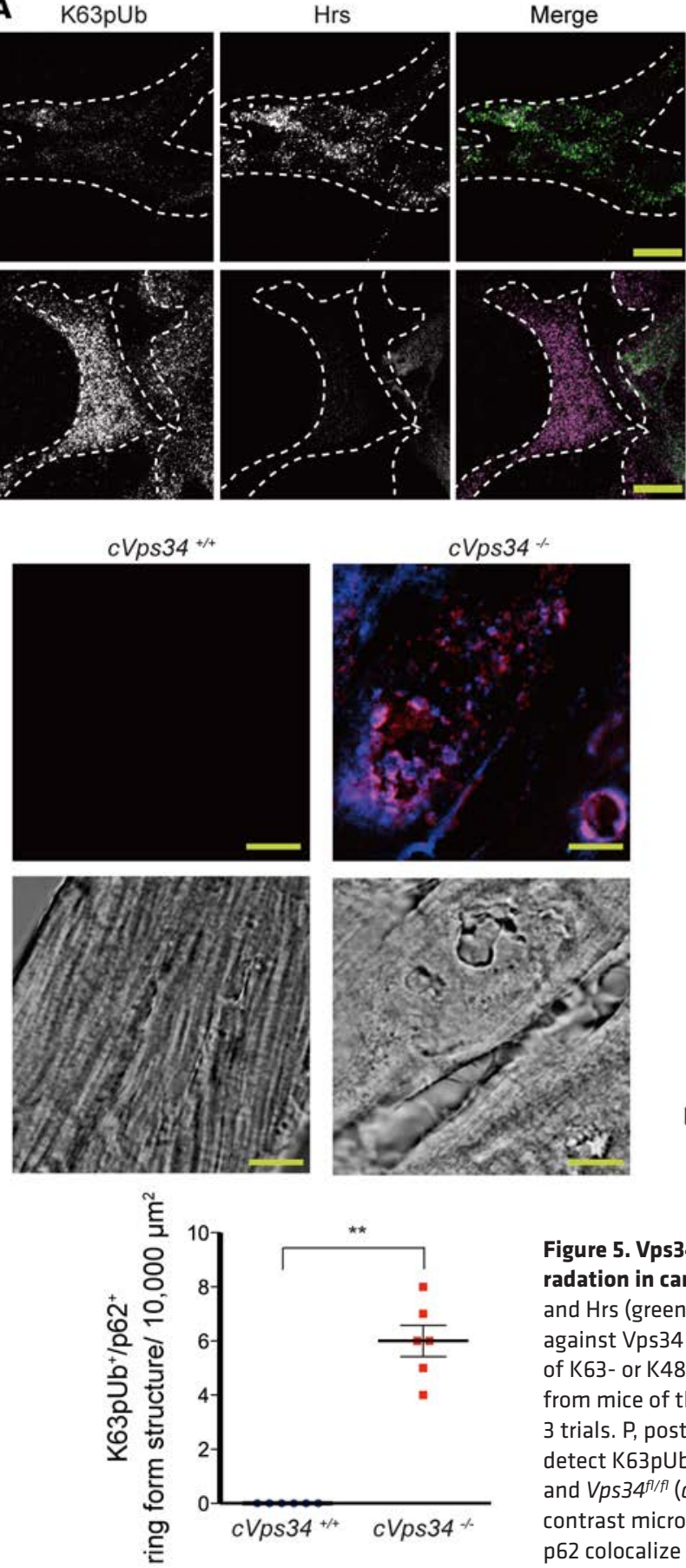

B
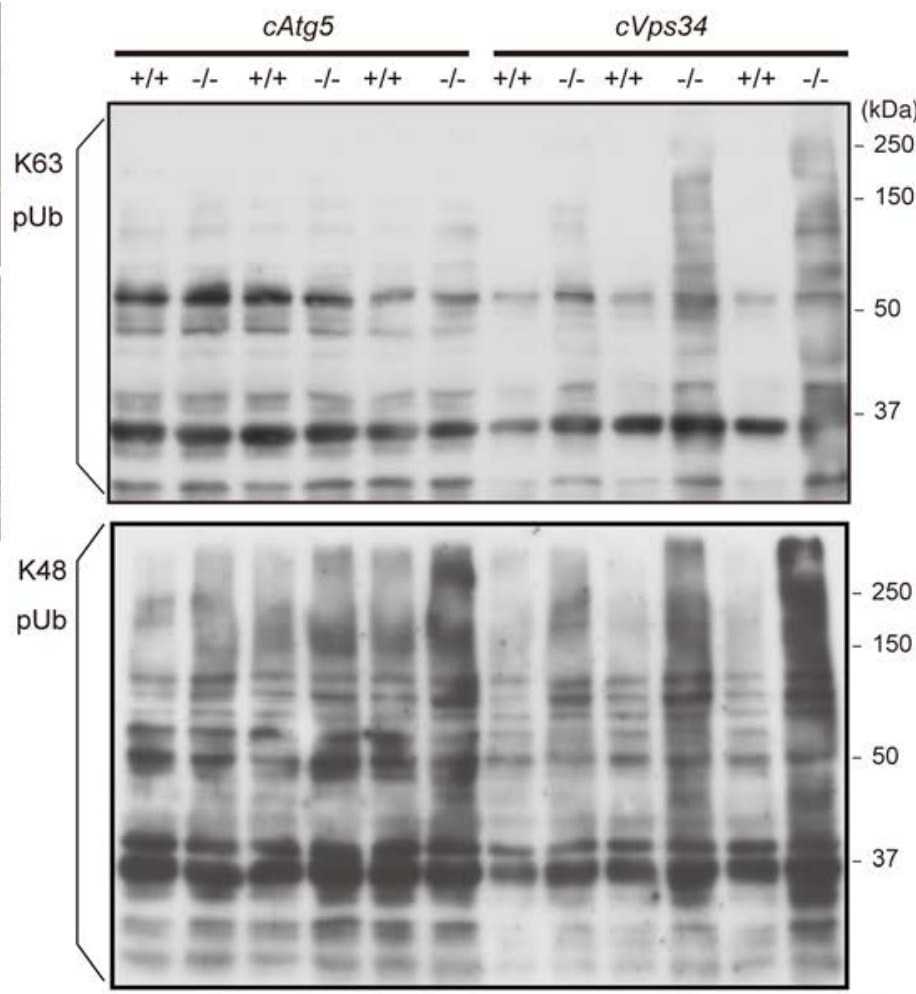

Atg 5

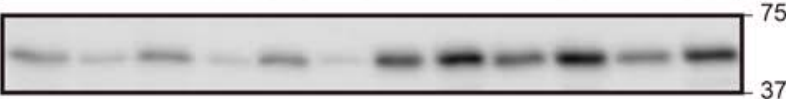

Vps34

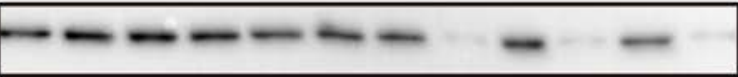

100

$\operatorname{Lamin} \mathrm{A}$

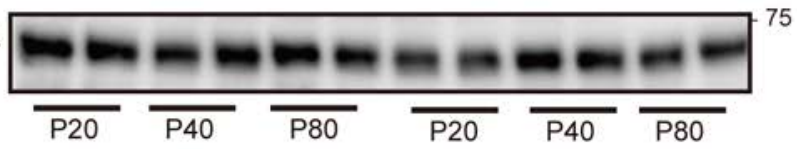

Figure 5. Vps34 is required for ESCRT-mediated K63-linked polyubiquitinated protein degradation in cardiomyocytes. (A) Immunocytochemistry to detect K63pUb proteins (magenta) and $\mathrm{Hrs}$ (green) in P19.CL6 cells transfected for 72 hours with control siRNA (Ctrl) or siRNA against Vps34 (siVps34). Scale bars: $10 \mu \mathrm{m}$. Results are representative of 3 trials. (B) Detection of K63- or K48-linked polyubiquitinated proteins in cardiac total membrane fractions prepared from mice of the indicated genotypes. Lamin, loading control. Results are representative of 3 trials. P, postnatal day. (C) Upper panels: Representative immunofluorescence staining to

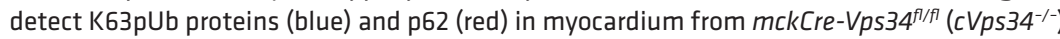
and $V p s 34^{f / f l}\left(c \vee p s 34^{+/+}\right)$mice at P80. Middle panels: Corresponding differential interference contrast microscopy images of the specimens are shown. Note that K63pUb proteins and p62 colocalize on ring-form structures in Vps34-deficient hearts. Scale bars: $10 \mu \mathrm{m}$. Lower panel: Quantification of K63pUb+/p62+ ring-form structures in cardiomyocytes. $\mathrm{K} 63 \mathrm{pUb} / \mathrm{p}^{+} / 2^{+}$ structures $\left(>5 \mu \mathrm{m}\right.$ in diameter) were counted in 5 randomly chosen fields $\left(10,000 \mu \mathrm{m}^{2}\right)$ for each sample. Data are the mean \pm SEM ( $n=6$ /group). ${ }^{*} P<0.01$, 2 -tailed Student's $t$ test.

transgenic mice, the PCR primers 5'-TTTGCCTGCATTACCGGTCGATGCAAC-3' and 5'-TGCCCCTGTTTCACTATCCAGGTTACGGA-3' were used to detect the Cre allele. Southern blot analysis was performed after digestion of cardiac genomic DNA with PstI. Vps34//+ mice were backcrossed 6-10 times onto the C57BL/6JJ background before intercrossing. To generate muscle-specific Vps34-deficient mice, backcrossed $V p s 34^{\text {I/fl }}$ mice were bred with $m c k$-Cre transgenic mice (42). Transgenic EEA1-FYVE-GFP mice were generated as described (43), except that an EEA1-FYVE sequence was inserted into pCAGGS, in which gene expression is controlled by the cytomegalovirus enhancer and chicken $\beta$-actin promoter. Atg $5^{\text {I/fl}}$ mice were generated as previously described (44). 
A

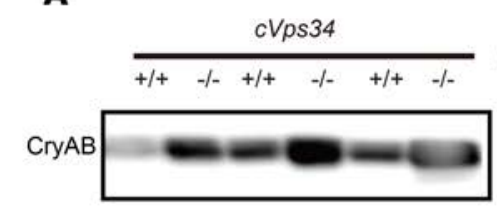

E
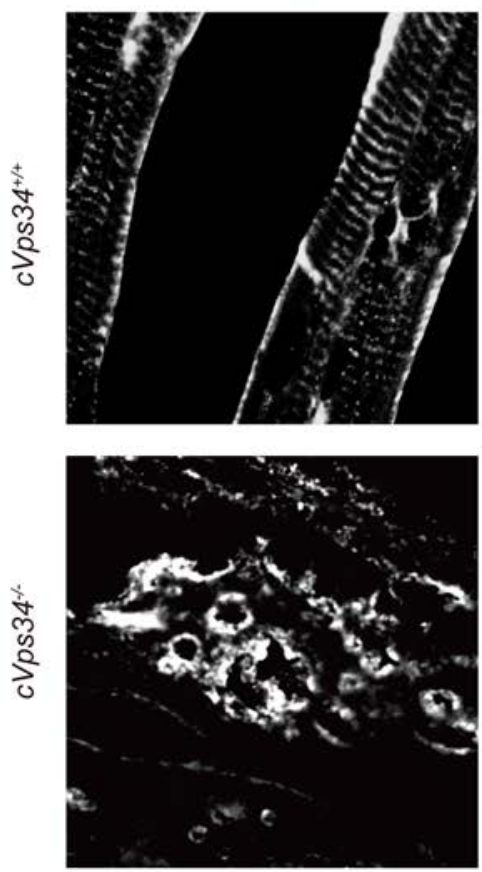

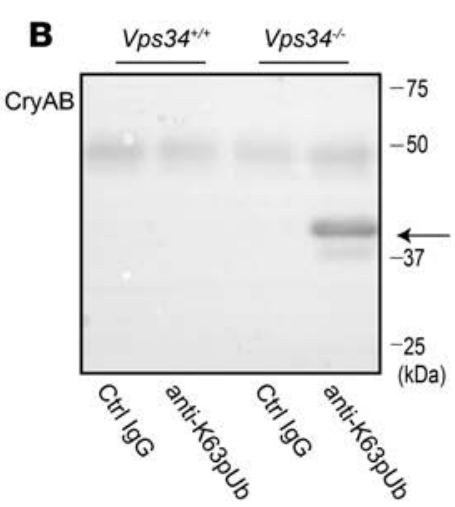

Des
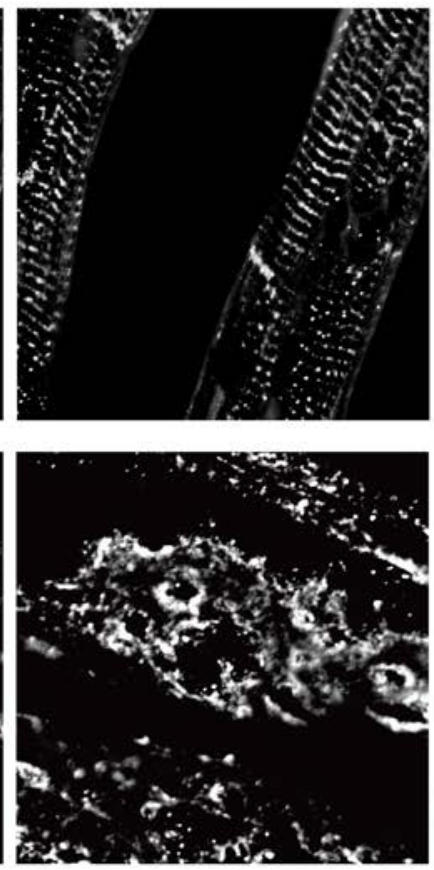

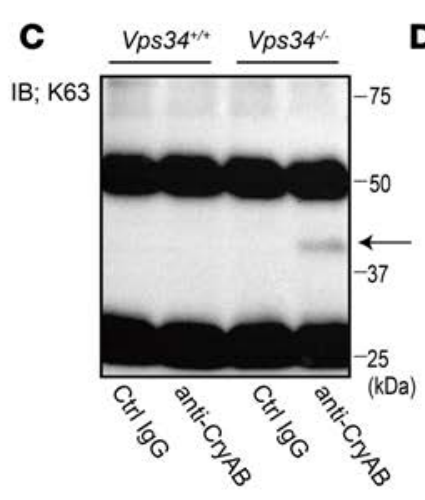

p62
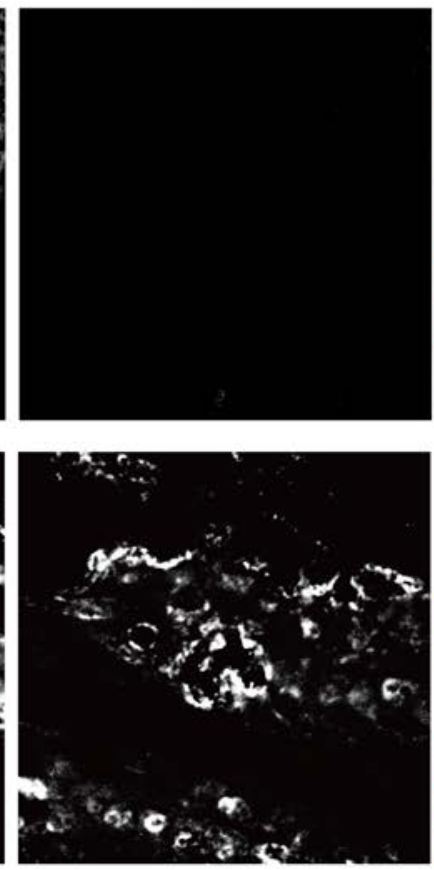

D IP; FLAG (Ub)

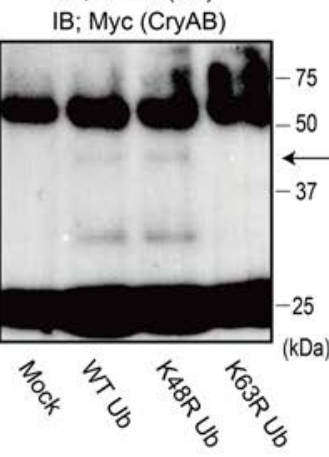

Merge

Figure 6. Loss of Vps34 abrogates degradation of K63-linked polyubiquitinated CryAB. (A) Immunoblot to detect CryAB in the NP-40-insoluble fraction of heart lysates from mckCre-Vps34//fl $\left(\mathrm{cVps} 34^{-/-}\right)$mice and littermate control $\left(c V p s 34^{+/+}\right)$at the indicated age. Lamin A, loading control. Results are representative of 3 trials. (B and C) Detection of K63-linked polyubiquitinated CryAB ( $40-\mathrm{kDa}$ bands; arrows) in the cardiac insoluble fractions from cVps34 $4^{--}$mice at P80 ( $\left.n=4\right)$. (B) Proteins were immunoprecipitated (IP) with anti-K63pUb (K63) Ab or control (Ctrl) IgC followed by immunoblotting (IB) with anti-CryAB Ab. (C) IP with anti-CryAB Ab or Ctrl IgC followed by IB with anti-K63pUb Abs. Results are representative of 3 trials. (D) Immunoblot to detect Myc-tagged CryAB in lysates of P19.CL6 cells that were mock transfected or transfected with Myc-tagged CryAB plus FLAG-tagged WT, K48R-, or K63R-ubiquitin. Ubiquitinated proteins were immunoprecipitated with anti-FLAC Ab and immunoblotted with anti-Myc Ab to detect Myctagged CryAB. Arrow, $\sim 40-\mathrm{kDa}$ band of ubiquitinated CryAB. Results are representative of 3 trials. (E) Immunofluorescence detection of CryAB (blue),

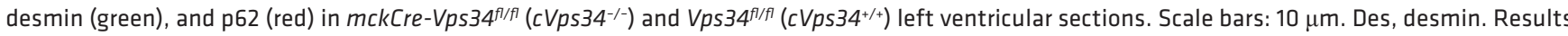
are representative of at least 10 specimens examined per group.

Cells. The P19.CL6 mouse embryonic carcinoma cell line was purchased from RIKEN Bio Resource Center. P19.CL6 cells were cultured in MEM- $\alpha$ containing 10\% fetal bovine serum and were induced to differentiate into cardiomyocyte-like cells by adding $1 \%$ DMSO to the culture medium. Differentiation of P19.CL6 cells was confirmed as previously described (45).

Mouse primary cardiomyocytes were isolated from cardiac left ventricular tissues using a collagenaseprotease technique as described previously (46).

RNA interference. siRNAs targeting Vps34/Pik3c3 and Hrs were obtained from Thermo Fisher Scientific (Stealth RNAi siRNA system). The sequences of each siRNA are shown below. Transfection was performed using the Neon Transfection System (Invitrogen) according to the manufacturer's protocol. Differentiated P19.CL6 cells were seeded into $10-\mathrm{cm}$ dishes at $1.0 \times 10^{7} \mathrm{cells} / \mathrm{dish}$, transfected with 

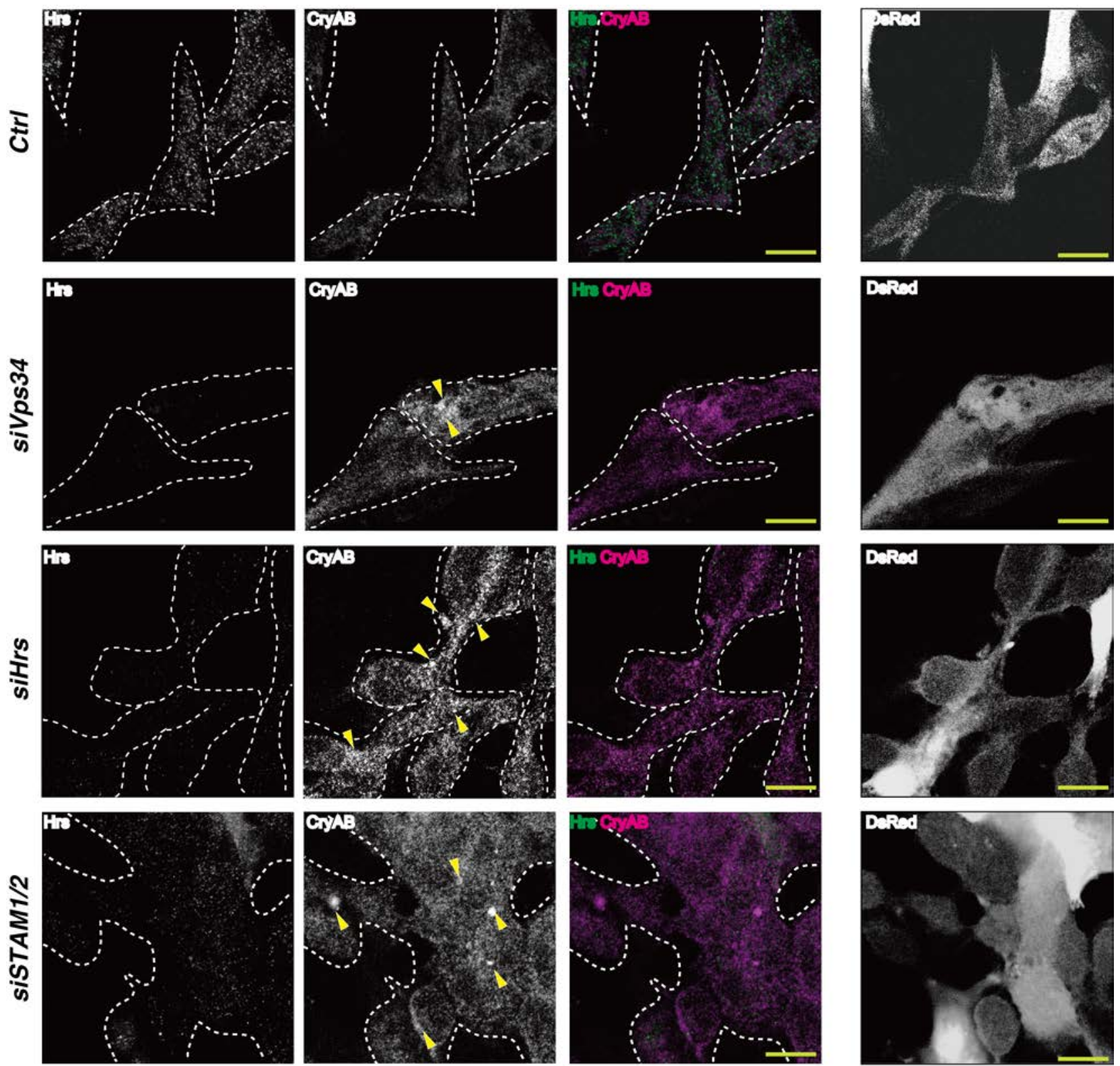

Figure 7. A Vps34-Hrs axis contributes to degradation of CryAB. Left panels: Immunocytochemistry to detect CryAB-positive aggregates in P19.CL6 cells transfected with control siRNA (Ctrl), siVps34, siHrs, or siSTAM1 and siSTAM2. Cells were immunostained with anti-CryAB (magenta) and antiHrs (green) Abs. Arrowheads indicate aggregates of CryAB. Right panels: Corresponding images of P19.CL6 cells cotransfected with a red fluorescent protein (DsRed). Results are representative of 3 trials. Scale bars: $10 \mu \mathrm{m}$.

DsRed expression vector and siVps34 or control siRNA. The cells were incubated for 72 hours at $37^{\circ} \mathrm{C}$. Effective suppression of the target protein expression by each siRNA was confirmed by immunoblotting and immunocytochemistry. The sequences of siRNAs are as follows: Vps34-1, GGGACCCAAAGACTCACGAGATGTA; Vps34-2, GGTTGATGCAAACATTCCAGATATT; Vps34-3， GCAGATCCATAAGTTTGCCCAGTAC; Hrs-1, TGGAGGTGCAGAGACAGCTAGCTAT; Hrs-2, GGAGTCAGACTGGGAGTCCATTCTA; Hrs-3， CCTGCTATGAGCAGCTGAACAAGAA; STAM1-1, CAGAACTGTCAGAACTAAACGTGAA; STAM2-1, CGGTTTGCCTCAGCTGGCTGGCTTT.

Flow cytometric analysis of autophagy. P19.CL6 cells cotransfected with GFP-LC3 and siRNAs were cultured in regular medium for 72 hours. For induction of autophagy, the cells were further incubated for 24 hours in amino acid-free MEM- $\alpha$ without serum. Total cellular GFP-LC3 signals were analyzed by flow cytometry (FACSCalibur, BD). 

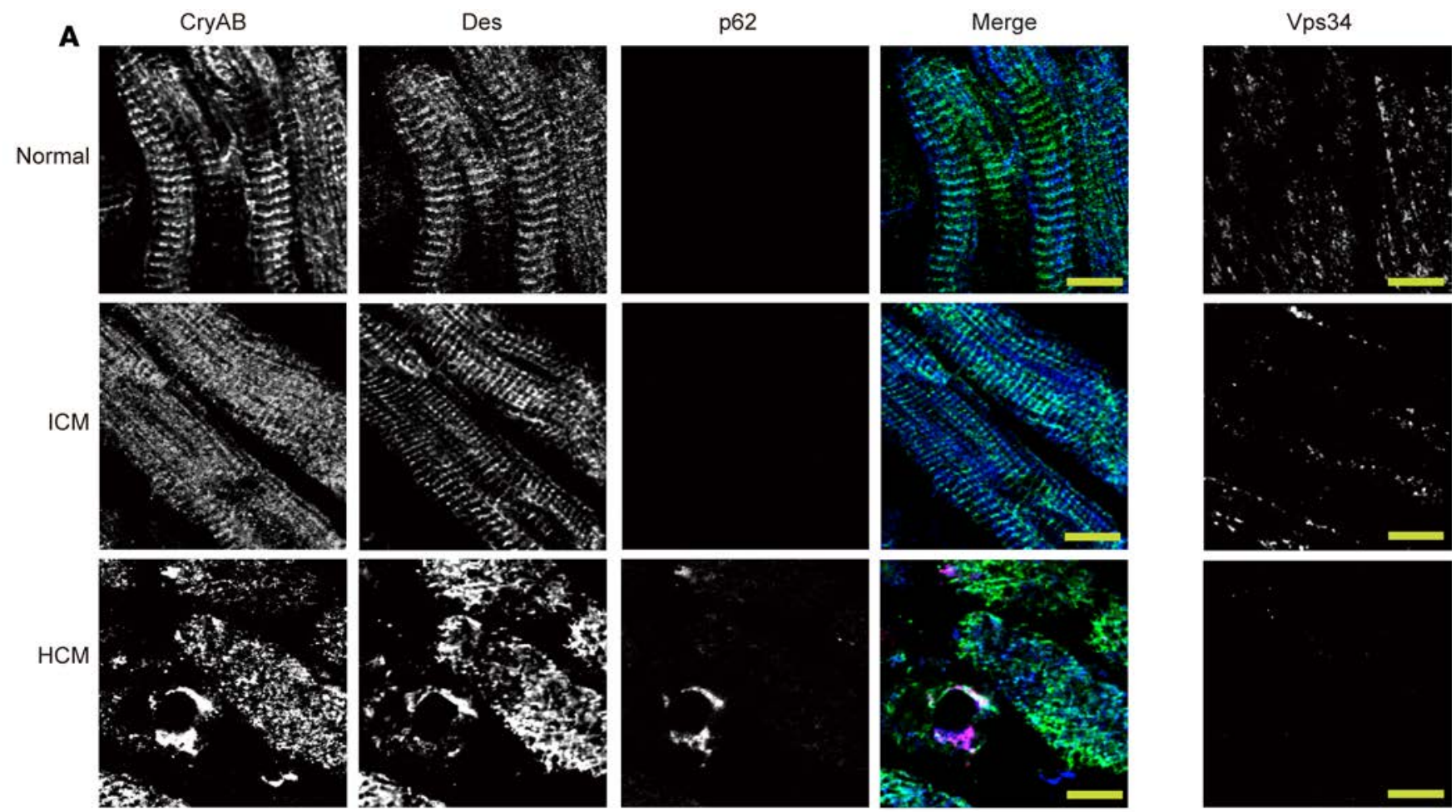

B

K63

p62

Vps34

Merge

DIC
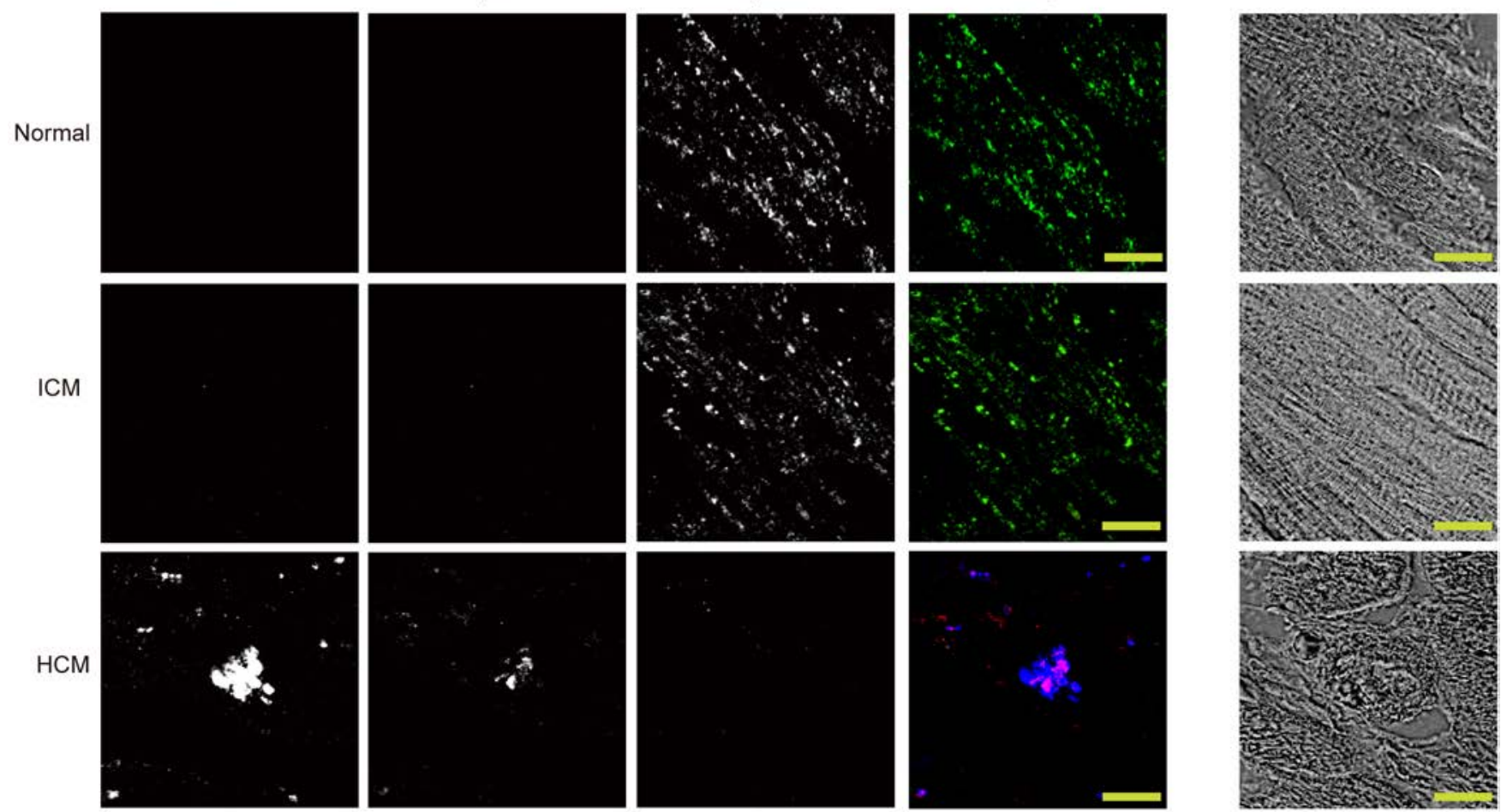

Figure 8. Aberrant accumulations of CryAB, desmin, K63-linked polyubiquitinated proteins, and p62 in human HCM. (A and B) Serial sections showing immunofluorescent staining of the indicated proteins in human LV specimens from a normal individual, an ICM patient, and a hypertrophic cardiomyopathy (HCM) patient. Scale bars: $10 \mu \mathrm{m}$. (A) Left panels: CryAB (blue); Des, desmin (green); p62 (red). Right panels: corresponding immunostaining for Vps34. (B) Left panels: K63, K63-pUb (blue); p62 (red); Vps34 (green). Right panels: Corresponding differential interference contrast microscopy images of the specimens are shown. 


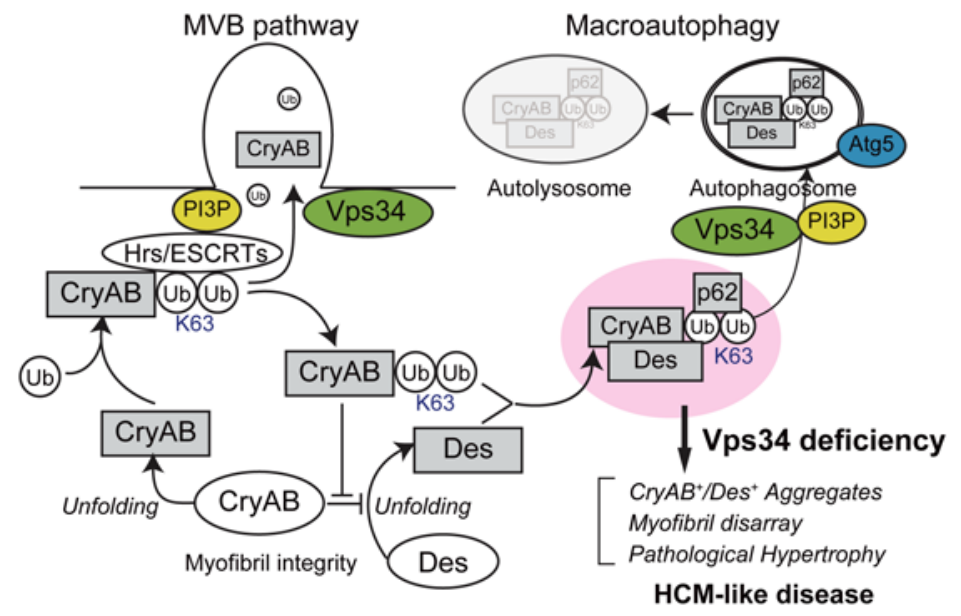

Figure 9. Schematic illustration of a proposed mechanism by which Vps34 deficiency might promote hypertrophic cardiomyopathy-like disease. K63, K63pUb; MVB, multivesicular body; Des, desmin; CryAB, $\alpha B$-crystallin; PI3P, phosphatidylinositol 3-phosphate; Ub, ubiquitin. For details, please see the Discussion in the main text.

Protein analyses. Excised 20-50 mg of left ventricular myocardium was frozen in liquid nitrogen and homogenized in an ice-cold lysis buffer containing $50 \mathrm{mM}$ Tris, $\mathrm{pH} 7.5$, $150 \mathrm{mM} \mathrm{NaCl}, 1 \% \mathrm{NP}-40$ and protease inhibitor cocktail (Roche). The supernatant was stored as the soluble fraction. The separated pellet was then resuspended in the lysis buffer containing $8 \mathrm{M}$ urea, and the resultant supernatant was kept as the insoluble fraction. $\mathrm{K} 63 \mathrm{pUb}$ and $\mathrm{CryAB}$ were immunoprecipitated with control IgG, $\mathrm{K} 63 \mathrm{pUb}$, or anti-CryAB

Abs from the insoluble fraction. Cytosolic and total membrane fractions of murine hearts and P19.CL6 cells were prepared using the Plasma Membrane Protein Extraction Kit (BioVision) according to the manufacturer's protocol. For immunoblotting, primary Abs were visualized using TrueBlot (Rockland) or HRPconjugated secondary Abs (GE Healthcare).

Echocardiography and electrocardiogram telemetry. Echocardiography was performed as described previously (47). Electrocardiogram telemetry was performed in P80 male mice that were anesthetized and implanted with a TA10ETA-F20 transmitter. All data were recorded and analyzed using the PhysioTel and Dataquest system (Data Sciences International).

Histological analyses, immunocytochemistry, and Abs. For standard histology, hearts were excised from anesthetized mice, fixed in PBS containing 10\% formaldehyde, and embedded in paraffin. Sections of $2 \mu \mathrm{m}$ were cut and subjected to standard Elastica-Masson staining, Gomori trichrome staining, or H\&E staining.

For electron microscopy, excised hearts were fixed overnight at $4^{\circ} \mathrm{C}$ in $0.1 \mathrm{M}$ cacodylate, $2 \%$ paraformaldehyde, and $2 \%$ glutaraldehyde in water. After incubation for 2 hours in $1 \% \mathrm{OsO}_{4}$ in $0.1 \mathrm{M}$ cacodylate buffer, the specimens were dehydrated in an ethanol series, passed through propylene oxide, and embedded in epoxy resin. Ultrathin sections $(60 \mathrm{~nm})$ were collected on copper grids and stained for 10 minutes in $4 \%$ uranyl acetate. The specimens were observed with a Hitachi H-7650 electron microscope.

For immunohistochemistry, frozen heart sections of $10 \mu \mathrm{m}$ thickness were cut and placed onto glass slides, followed by $5 \%$ normal goat serum, and incubated with primary $\mathrm{Abs}$ (see below) at $4^{\circ} \mathrm{C}$ overnight. Slides were then incubated with the appropriate secondary Abs at room temperature (RT) for 30 min utes before analysis by confocal fluorescence microscopy using a LSM510META Ver3.2 microscope (Carl Zeiss). For quantification, $\mathrm{CryAB}^{+} / \mathrm{Des}^{+}$aggregate $(>5 \mu \mathrm{m}$ in diameter) were counted in 5 randomly chosen fields $\left(10,000 \mu \mathrm{m}^{2}\right)$ for each sample.

Antibodies. Primary Abs for immunoblotting (IB), immunoprecipitation (IP), and immunofluorescent staining (IF) were as follows: anti-Vps34, rabbit polyclonal, catalog 3811 and clone D9A5 (IB, 1:1,000 dilution, Cell Signaling Technology); anti-Vps34, clone EPR5301 (IF, 1:100, Epitomics/Abcam); antiAtg5, clone EPR4797 (IB, 1:1,000, Epitomics/Abcam); anti-ubiquitin, Lys63-specific, clone Apu3 (IP, 1:50, Millipore); anti-ubiquitin, Lys63-specific, clone HWA4C4 (IF, 1:50, Millipore); anti-ubiquitin, Lys63specific, clone D7A11 (IB, 1:1,000, Cell Signaling Technology); anti-ubiquitin, Lys48-specific, clone Apu2 (IP, 1:100; IF, 1:100, Millipore); anti-ubiquitin, Lys48-specific, clone D9D5 (IB, 1:1,000, Cell Signaling Technology); anti-p62 rabbit polyclonal, catalog PM045 (IB, 1:1,000; IP, 1:100, MBL); anti-p62 guinea pig polyclonal, catalog PM066 (IF, 1:100, MBL); anti-LC3 rabbit polyclonal, catalog PM036, (IB, 1:1,000; IF, 1:100, MBL); anti-desmin, clone D93F5 (IB, 1:1,000; IF frozen section, 1:100, Cell Signaling Technology); anti-desmin polyclonal, catalog ab86083 (IF paraffin-embedded section, 1:100, Abcam), $\alpha \mathrm{B}$-crystallin, 1B6.1-3G4 (IB, 1:1,000; IF frozen section, 1:100; IF paraffin-embedded section, 1:100, Enzo Biosciences); anti-Hrs, rabbit polyclonal, catalog SAB4503560 (IB, 1:1,000; IF, 1:100, Sigma-Aldrich) and clone D7T5N (IB, 1:1,000, Cell Signaling Technology); anti-phospho- and total mTor, anti-phospho- and total 4EBP1, anti-phospho- and total S6, (clone D9C2, 7C10, 236B4, 53H11, D57.2.2E, and 5G10, respectively) (IB, 1:1,000, Cell Signaling Technology); and anti-GAPDH, clone 6C5 (IB, 1:2,000, Millipore). Tissues were 
lysed in homogenization buffer alone $(50 \mathrm{mM}$ Tris- $\mathrm{HCl}, \mathrm{pH} 7.5,150 \mathrm{mM} \mathrm{NaCl}, 1 \% \mathrm{NP}-40$ and protease inhibitor cocktail [Roche]), or in homogenization buffer containing $8 \mathrm{M}$ urea plus protease inhibitor cocktail. Primary Abs were visualized using secondary Abs (GE Healthcare) and ImmunoStar Reagents (WAKO) or SuperSignal West Pico Chemiluminescent Substrate (Thermo Fisher Scientific).

Study approval. All investigations involving human tissue in our study were approved by the Ethics Committee of Akita University Graduate School of Medicine and the Faculty of Medicine (Akita-city, Akita, Japan). All experiments in animals were approved by the Laboratory Animal Research Committee of the Biomedical Education Research Center of Akita University (Akita-city, Akita, Japan), and were performed in accordance with the institutional guidelines.

Statistics. Statistical analyses were performed using GraphPad Prism software, version 5 . Results are the mean \pm SEM or the median value. Paired data were evaluated by the 2-tailed Student's $t$ test and MannWhitney $U$ test, whereas ANOVA and the Kruskal-Wallis test were used for comparisons of multiple variables. Survival curves were plotted using the Kaplan-Meier method and evaluated using the log-rank test. A $P$ value of less than 0.05 was considered statistically significant.

\section{Author contributions}

HK, JS, and TS initiated the project and designed the research plan. JS, AS, and NM generated genetargeted mice. KK and YI performed electrocardiogram telemetry measurements. HK conducted most experiments and analyzed data. SE, ST, HN, and MY performed mouse experiments. HW, HI, and AG made clinical and histological diagnoses. HK and TS wrote the manuscript.

\section{Acknowledgments}

The authors thank H. Kurose for cardiomyocyte culture; S. Chida and H. Watanabe for tissue processing; and K. Kofuji, At. Kato, Ak. Kato, S. Kumagai, Y. Kadowaki, Y. Sugihara, T. Ayukawa, K. Asanuma, C. Horie, Y. Horie, Y. Moribayashi, S. Nishino, Y. Kuribayashi, S. Ooi, and K. Yanase for critical discussions and technical support. This work was supported in part by research grants from the Japan Science and Technology Agency (JST); the Ministry of Education, Culture, Sports, and Technology of Japan; the Japan Society for the Promotion of Science (JSPS) (JSPS KAKENHI grants JP24790734, JP26253006, JP15H05899, and JP15H0589711); the Japan Agency for Medical Research and Development (AMED) (grant 16gm0710002h0304); the Ono Medical Research Foundation; and the Daiichi Sankyo Foundation of Life Science.

Address correspondence to: Takehiko Sasaki, 1-1-1 Hondo, 010-8543, Akita, Japan. Phone: 81.18.884.6080 or 81.18.884.6079; E-mail: tsasaki@med.akita-u.ac.jp.

1. Ezzati M, Obermeyer Z, Tzoulaki I, Mayosi BM, Elliott P, Leon DA. Contributions of risk factors and medical care to cardiovascular mortality trends. Nat Rev Cardiol. 2015;12(9):508-530.

2. Authors/Task Force members, et al. 2014 ESC Guidelines on diagnosis and management of hypertrophic cardiomyopathy: the Task Force for the Diagnosis and Management of Hypertrophic Cardiomyopathy of the European Society of Cardiology (ESC). Eur Heart J. 2014;35(39):2733-2779.

3. Maron BJ, Maron MS. Hypertrophic cardiomyopathy. Lancet. 2013;381(9862):242-255.

4. Marian AJ. Hypertrophic cardiomyopathy: from genetics to treatment. Eur J Clin Invest. 2010;40(4):360-369.

5. Funada A, et al. Heterogeneity of clinical manifestation of hypertrophic cardiomyopathy caused by deletion of lysine 183 in cardiac troponin I gene. Int Heart J. 2010;51(3):214-217.

6. Seiler C, Jenni R, Vassalli G, Turina M, Hess OM. Left ventricular chamber dilatation in hypertrophic cardiomyopathy: related variables and prognosis in patients with medical and surgical therapy. Br Heart J. 1995;74(5):508-516.

7. Goldfarb LG, Dalakas MC. Tragedy in a heartbeat: malfunctioning desmin causes skeletal and cardiac muscle disease. $J$ Clin Invest. 2009;119(7):1806-1813.

8. Dalakas MC, Park KY, Semino-Mora C, Lee HS, Sivakumar K, Goldfarb LG. Desmin myopathy, a skeletal myopathy with cardiomyopathy caused by mutations in the desmin gene. N Engl J Med. 2000;342(11):770-780.

9. Vicart P, et al. A missense mutation in the alphaB-crystallin chaperone gene causes a desmin-related myopathy. Nat Genet. 1998;20(1):92-95.

10. Bär H, Strelkov SV, Sjöberg G, Aebi U, Herrmann H. The biology of desmin filaments: how do mutations affect their structure, assembly, and organisation? J Struct Biol. 2004;148(2):137-152.

11. Sun Y, MacRae TH. The small heat shock proteins and their role in human disease. FEBS J. 2005;272(11):2613-2627.

12. Sanbe A, et al. Desmin-related cardiomyopathy in transgenic mice: a cardiac amyloidosis. Proc Natl Acad Sci USA. 2004;101(27):10132-10136.

13. Kramer CM, et al. Hypertrophic Cardiomyopathy Registry: The rationale and design of an international, observational study of 
hypertrophic cardiomyopathy. Am Heart J. 2015;170(2):223-230.

14. Wang C, Wang X. The interplay between autophagy and the ubiquitin-proteasome system in cardiac proteotoxicity. Biochim Biophys Acta. 2015;1852(2):188-194.

15. Herman PK, Emr SD. Characterization of VPS34, a gene required for vacuolar protein sorting and vacuole segregation in Saccharomyces cerevisiae. Mol Cell Biol. 1990;10(12):6742-6754.

16. Sasaki T, et al. Mammalian phosphoinositide kinases and phosphatases. Prog Lipid Res. 2009;48(6):307-343

17. Mizushima N, Komatsu M. Autophagy: renovation of cells and tissues. Cell. 2011;147(4):728-741.

18. Rusten TE, Vaccari T, Stenmark H. Shaping development with ESCRTs. Nat Cell Biol. 2011;14(1):38-45.

19. Jaber N, et al. Class III PI3K Vps34 plays an essential role in autophagy and in heart and liver function. Proc Natl Acad Sci USA. 2012;109(6):2003-2008.

20. Kawamura N, et al. Delivery of endosomes to lysosomes via microautophagy in the visceral endoderm of mouse embryos. Nat Commun. 2012;3:1071.

21. Elliott P, et al. Classification of the cardiomyopathies: a position statement from the European Society Of Cardiology Working Group on Myocardial and Pericardial Diseases. Eur Heart J. 2008;29(2):270-276.

22. McLendon PM, Robbins J. Desmin-related cardiomyopathy: an unfolding story. Am J Physiol Heart Circ Physiol. 2011;301(4):H1220-H1228.

23. Tooze SA, Yoshimori T. The origin of the autophagosomal membrane. Nat Cell Biol. 2010;12(9):831-835.

24. Duncan LM, et al. Lysine-63-linked ubiquitination is required for endolysosomal degradation of class I molecules. $E M B O J$. 2006;25(8):1635-1645.

25. Lauwers E, Jacob C, André B. K63-linked ubiquitin chains as a specific signal for protein sorting into the multivesicular body pathway. J Cell Biol. 2009;185(3):493-502.

26. Morishita H, et al. Deletion of autophagy-related 5 (Atg5) and Pik3c3 genes in the lens causes cataract independent of programmed organelle degradation. J Biol Chem. 2013;288(16):11436-11447.

27. Perng MD, et al. The cardiomyopathy and lens cataract mutation in alphaB-crystallin alters its protein structure, chaperone activity, and interaction with intermediate filaments in vitro. J Biol Chem. 1999;274(47):33235-33243.

28. Koh TJ, Escobedo J. Cytoskeletal disruption and small heat shock protein translocation immediately after lengthening contractions. Am J Physiol, Cell Physiol. 2004;286(3):C713-C722.

29. Zheng Q, Su H, Ranek MJ, Wang X. Autophagy and p62 in cardiac proteinopathy. Circ Res. 2011;109(3):296-308.

30. Sciarretta S, Volpe M, Sadoshima J. Mammalian target of rapamycin signaling in cardiac physiology and disease. Circ Res. 2014;114(3):549-564.

31. Schlossarek S, Frey N, Carrier L. Ubiquitin-proteasome system and hereditary cardiomyopathies. J Mol Cell Cardiol. 2014;71:25-31.

32. Tannous $\mathrm{P}$, et al. Autophagy is an adaptive response in desmin-related cardiomyopathy. Proc Natl Acad Sci USA. 2008;105(28):9745-9750.

33. Liu XM, Sun LL, Hu W, Ding YH, Dong MQ, Du LL. ESCRTs cooperate with a selective autophagy receptor to mediate vacuolar targeting of soluble cargos. Mol Cell. 2015;59(6):1035-1042.

34. Sahu R, et al. Microautophagy of cytosolic proteins by late endosomes. Dev Cell. 2011;20(1):131-139.

35. Taelman VF, et al. Wnt signaling requires sequestration of glycogen synthase kinase 3 inside multivesicular endosomes. Cell. 2010;143(7):1136-1148.

36. Parkinson N, et al. ALS phenotypes with mutations in CHMP2B (charged multivesicular body protein 2B). Neurology. 2006;67(6):1074-1077.

37. Skibinski G, et al. Mutations in the endosomal ESCRTIII-complex subunit CHMP2B in frontotemporal dementia. Nat Genet. 2005;37(8):806-808.

38. Tan JM, et al. Lysine 63-linked ubiquitination promotes the formation and autophagic clearance of protein inclusions associated with neurodegenerative diseases. Hum Mol Genet. 2008;17(3):431-439.

39. Bova MP, et al. Mutation R120G in alphaB-crystallin, which is linked to a desmin-related myopathy, results in an irregular structure and defective chaperone-like function. Proc Natl Acad Sci USA. 1999;96(11):6137-6142.

40. Haley DA, Horwitz J, Stewart PL. The small heat-shock protein, alphaB-crystallin, has a variable quaternary structure. $J M o l$ Biol. 1998;277(1):27-35.

41. Kofuji S, et al. INPP4B is a PtdIns(3,4,5)P3 phosphatase that can act as a tumor suppressor. Cancer Discov. 2015;5(7):730-739.

42. Brüning JC, et al. A muscle-specific insulin receptor knockout exhibits features of the metabolic syndrome of NIDDM without altering glucose tolerance. Mol Cell. 1998;2(5):559-569.

43. Nishio M, et al. Control of cell polarity and motility by the PtdIns(3,4,5)P3 phosphatase SHIP1. Nat Cell Biol. 2007;9(1):36-44.

44. Hara T, et al. Suppression of basal autophagy in neural cells causes neurodegenerative disease in mice. Nature. 2006;441(7095):885-889.

45. Clement CA, et al. The primary cilium coordinates early cardiogenesis and hedgehog signaling in cardiomyocyte differentiation J Cell Sci. 2009;122(Pt 17):3070-3082.

46. Hoppe UC, Johns DC, Marbán E, O’Rourke B. Manipulation of cellular excitability by cell fusion: effects of rapid introduction of transient outward $\mathrm{K}^{+}$current on the guinea pig action potential. Circ Res. 1999;84(8):964-972.

47. Kuba K, et al. Impaired heart contractility in Apelin gene-deficient mice associated with aging and pressure overload. Circ Res 2007;101(4):e32-e42. 